\title{
Maternal Experience-Dependent Cortical Plasticity in Mice Is Circuit- and Stimulus-Specific and Requires MECP2
}

\author{
BBilly Y.B. Lau, ${ }^{-K e e r t h i ~ K r i s h n a n, ~ Z . ~ J o s h ~ H u a n g, ~ a n d ~} @$ Stephen D. Shea \\ Cold Spring Harbor Laboratory, Cold Spring Harbor, New York 11724
}

The neurodevelopmental disorder Rett syndrome is caused by mutations in the gene Mecp2. Misexpression of the protein MECP2 is thought to contribute to neuropathology by causing dysregulation of plasticity. Female heterozygous Mecp 2 mutants (Mecp $2^{\text {het }}$ ) failed to acquire a learned maternal retrieval behavior when exposed to pups, an effect linked to disruption of parvalbumin-expressing inhibitory interneurons (PV) in the auditory cortex. Nevertheless, how dysregulated PV networks affect the neural activity dynamics that underlie auditory cortical plasticity during early maternal experience is unknown. Here we show that maternal experience in WT adult female mice (WT) triggers suppression of PV auditory responses. We also observe concomitant disinhibition of auditory responses in deep-layer pyramidal neurons that is selective for behaviorally relevant pup vocalizations. These neurons further exhibit sharpened tuning for pup vocalizations following maternal experience. All of these neuronal changes are abolished in $M e c p 2^{\text {het }}$, suggesting that they are an essential component of maternal learning. This is further supported by our finding that genetic manipulation of GABAergic networks that restores accurate retrieval behavior in $M e c p 2^{\text {het }}$ also restores maternal experience-dependent plasticity of PV. Our data are consistent with a growing body of evidence that cortical networks are particularly vulnerable to mutations of Mecp 2 in PV neurons. Moreover, our work links, for the first time, impaired in vivo cortical plasticity in awake Mecp2 mutant animals to a natural, ethologically relevant behavior.

Key words: auditory cortex; maternal behavior; Mecp2; neurophysiology; parvalbumin; plasticity

Significance Statement

Rett syndrome is a genetic disorder that includes language communication problems. Nearly all Rett syndrome is caused by mutations in the gene that produces the protein MECP2, which is important for changes in brain connectivity believed to underlie learning. We previously showed that female Mecp2 mutants fail to learn a simple maternal care behavior performed in response to their pups' distress cries. This impairment appeared to critically involve inhibitory neurons in the auditory cortex called parvalbumin neurons. Here we record from these neurons before and after maternal experience, and we show that they adapt their response to pup calls during maternal learning in nonmutants, but not in mutants. This adaptation is partially restored by a manipulation that improves learning.

\section{Introduction}

In humans, loss-of-function mutations in the gene MECP2 cause the neurodevelopmental disorder Rett syndrome (Amir et al., 1999). MECP2 protein binds to methylated DNA (Lewis et al., 1992) and controls transcriptional programs by modifying chro-

\footnotetext{
Received Aug. 13, 2019; revised Dec. 12, 2019; accepted Dec. 20, 2019.

Author contributions: B.Y.B.L., K.K., and S.D.S. designed research; B.Y.B.L. and K.K. performed research; B.Y.B.L., K.K., and S.D.S. analyzed data; B.Y.B.L., K.K., Z.J.H., and S.D.S. edited the paper; B.Y.B.L., K.K., and S.D.S. wrote the paper; Z.J.H. contributed unpublished reagents/analytic tools.

This work was supported by Simons Foundation Autism Research Initiative, Feil Family Foundation, and National Institute of Mental Health R01MH106656 to S.D.S., National Institute of Mental Health R01MH102616 to Z.J.H., and National Alliance for Research on Schizophrenia and Depression Young Investigator Grant, and the Brain and Behavior Research Foundation to K.K., and International Rett Syndrome Foundation Postdoctoral Fellowships to B.Y.B.L. and K.K. This work was also supported by University of Tennessee-Knoxville laboratory startup funds to K.K. We thank Jim Hall, Rebecca Prosser, and Robert Liu for helpful comments and discussion.

The authors declare no competing financial interests.

Correspondence should be addressed to Stephen D. Shea at sshea@cshl.edu.
}

matin structure (Skene et al., 2010; Agarwal et al., 2011; Brink et al., 2013; Maxwell et al., 2013). Most humans with Rett syndrome are females with a heterozygous loss-of-function mutation who are therefore mosaic for the gene (Van den Veyver and Zoghbi, 2000). Data from mice carrying mutations that abolish expression of MECP2 strongly suggest that it regulates plasticity in development and adulthood (Deng et al., 2010, 2014; McGraw et al., 2011; Noutel et al., 2011; Na et al., 2013; He et al., 2014; Krishnan et al., 2015, 2017; Tai et al., 2016; Gulmez Karaca et al., 2018). Nevertheless, how these mutations affect in vivo neuro-

B.Y.B. Lau's present address: Department of Biochemistry \& Cellular and Molecular Biology, University of University of Tennessee-Knoxville, Knoxville, TN 39996.

K. Krishnan's present address: Department of Biochemistry \& Cellular and Molecular Biology, University of University of Tennessee-Knoxville, Knoxville, TN 39996.

https://doi.org/10.1523/JNEUROSCI.1964-19.2019

Copyright $\odot 2020$ the authors 
physiology (Durand et al., 2012; Banerjee et al., 2016) and the plasticity that underlies learning are poorly understood.

MECP2 is expressed in all cells in the brain, yet parvalbuminexpressing cortical inhibitory interneurons (PV) appear to be especially vulnerable to loss of MECP2 (Ito-Ishida et al., 2015; He et al., 2017; Morello et al., 2018). Selective KO of Mecp2 in either $\mathrm{PV}$ or forebrain inhibitory neurons broadly recapitulates major phenotypes seen in whole-mouse KOs (Chao et al., 2010; ItoIshida et al., 2015). Among other functions (Cardin et al., 2009; Sohal et al., 2009; Atallah et al., 2012; Lee et al., 2012; Wilson et al., 2012; Kim et al., 2016), PV are crucial for regulating learning and plasticity during development and adulthood (Donato et al., 2013). Specifically, modulation of PV networks opens and closes windows of heightened plasticity, such as developmental critical periods (Levelt and Hübener, 2012; Takesian and Hensch, 2013). Therefore, dysregulation of PV in Mecp2 mutant mice disrupts developmental and adult learning (Picard and Fagiolini, 2019).

We recently showed that Mecp2 mutations impair auditory cortical plasticity and maternal vocal perception in adult female mice by altering PV (Krishnan et al., 2017). Primiparous female mice, or virgin females exposed to pups (surrogates), both exhibit experience-dependent improvement in retrieving isolated pups in response to their ultrasonic distress cries (Sewell, 1970; Ehret et al., 1987). Moreover, exposure of WT surrogates to pups triggers changes to inhibitory networks in the auditory cortex that affect responses to pup vocalizations (Liu and Schreiner, 2007; Galindo-Leon et al., 2009; Cohen et al., 2011; Lin et al., 2013; Cohen and Mizrahi, 2015; Marlin et al., 2015). Interestingly, we observed additional changes to PV in the auditory cortex of heterozygous Mecp2 mutants ( $\mathrm{MeCP} 2^{\text {het }}$ ), namely, increased expression of $\mathrm{PV}$ and intensification of perineuronal nets (PNNs). PNNs are extracellular matrix structures surrounding PV that act as physical barriers to synaptic modifications and control maturation of PV (Krishnan et al., 2015; Sorg et al., 2016; Miyata and Kitagawa, 2017; Sigal et al., 2019). Thus, PNNs are proposed to work in concert with elevated PV to act as "brakes" on plasticity that terminate critical periods (Takesian and Hensch, 2013). Indeed, genetic reduction of levels of the GABA-synthesizing enzyme GAD67 in the $\mathrm{MeCP} 2^{\text {het }}$ background normalized PV and PNN expression and restored maternal learning (Krishnan et al., 2017). Together, these findings predict that sensory responses to pup vocalizations in WT females are reshaped by PV following maternal experience, and that this plasticity is disrupted in $\mathrm{MeCP} 2^{\text {het }}$.

We made single-neuron recordings from awake, head-fixed mice to show that maternal experience in WT females (WT) triggers reduced inhibition and increased excitation in the auditory responses of presumptive deep-layer pyramidal neurons. Direct recordings from putative $\mathrm{PV}(\mathrm{pPV})$ neurons show decreased stimulus-evoked spiking following maternal experience. Therefore, changes in pyramidal cells may reflect disinhibition by the PV neurons. These changes are selective for behaviorally relevant pup vocalizations over synthetic stimuli, and pyramidal neurons also exhibit sharpened tuning for pup vocalizations following maternal experience. None of these neuronal changes was observed in $M e c p 2^{\text {het }}$. Indeed, in these mice, we found that $\mathrm{pPV}$ neuron output was increased in surrogates. Notably, the same GAD67 perturbation that restored retrieval behavior also reinstated $\mathrm{pPV}$ neuron plasticity following maternal experience.

We propose that: (1) MECP2 acts in cortical PV interneurons to coordinate stimulus-specific, experience-dependent cortical plasticity, thereby reshaping the output of deep layer pyramidal neurons; (2) mutations in Mecp2 disrupt cortical activity by ar- resting modulation of cortical PV networks; and (3) this has negative behavioral consequences.

\section{Materials and Methods}

Animals. Adult female mice (7-10 weeks old) were maintained on a 12 $\mathrm{h} / 12 \mathrm{~h}$ light-dark cycle (lights on 07:00 h) and received food ad libitum. Genotypes used were $\mathrm{CBA} / \mathrm{CaJ}, \mathrm{MeCP} 2^{\text {het }}$ (C57BL/6 background;

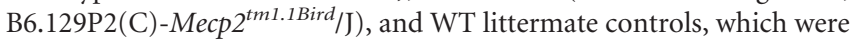
of the same genetic background as the mutants, but we refer to here as simply $W T$ for clarity and readability. Heterozygous female Ai32; PV-iresCre mice $\left(\mathrm{Ai}_{32}{ }^{+/-} ; \mathrm{PV}-\mathrm{Cre}^{+/-}\right)$were generated by crossing female Ai32 (B6;129S-Gt(ROSA)26Sor $\left.{ }^{\text {tm } 32(C A G-C O P 4 * H 134 R / E Y F P) H z e} / \mathrm{J}\right)$ and male PVires-Cre (B6;129P2-Pvalbtm1(cre)Arbr/J). The double-mutant Mecp2 $2^{\text {het }}$; Gad1 $^{\text {het }}$ (Het; Gad1 ${ }^{\text {het }}$ ) was generated by crossing Mecp $2^{\text {het }}$ females and $\mathrm{Gadl}^{\text {het }}$ males. Details on the Het; Gad1 ${ }^{\text {het }}$ line can be found in Krishnan et al. (2017). All procedures were conducted in accordance with the National Institutes of Health's Guide for the care and use of laboratory animals and approved by the Cold Spring Harbor Laboratory Institutional Animal Care and Use Committee.

Pup retrieval behavior. Pup retrieval behavior was performed as previously described (Krishnan et al., 2017). Briefly, 2 virgin female mice (1 $\mathrm{MeCP} 2^{\text {het }}$ and $1 \mathrm{WT}$ ) were cohoused with pregnant CBA/CaJ female 3-5 $\mathrm{d}$ before birth. Pup retrieval behavior was assessed starting on the day the pups were born (postnatal day 0). Pup retrieval assays were performed in these adult surrogates starting on the day the pups were born (postnatal day 0 ) as follows: (1) one surrogate was habituated with 3-5 pups in the nest of the home cage for $5 \mathrm{~min}$; (2) pups were removed for $2 \mathrm{~min}$; and (3) 1 pup was placed at each corner and 1 was placed in the center of the cage. The nest was left empty if there were $<5$ pups. Each surrogate was given a maximum of 5 min to retrieve all pups. Mothers, surrogates, and pups were returned to home cage after testing. Additional behavior trials were performed on days 3 and 5 . All behavioral assays were performed in the dark, during the light cycle between 10:30 AM and 4:00 PM and were recorded to video.

Surgery for awake recordings. We anesthetized the mice with a mixture of ketamine $(100 \mathrm{mg} / \mathrm{ml})$ and xylazine $(5 \mathrm{mg} / \mathrm{ml})$ and maintained with isoflurane. We affixed a head bar to the skull above the cerebellum using Rely X cement (3M) and methyl methacrylate-based dental cement (Teets). For additional stability, we secured 5 machine screws (Amazon Supply) to the skull before the application of cement. Animals were allowed to recover $>24 \mathrm{~h}$ before being used for recordings. For surrogate mice, surgery was performed immediately after behavioral assessment on day 5 , and recordings were obtained between days 7 and 11 .

Electrophysiology. On the day of recording, mice were reanesthetized with isoflurane and a craniotomy was made to expose the left hemisphere of the auditory cortex. Mice were then head-fixed via the attached head bar over a Styrofoam ball that was suspended above the air table. The Styrofoam ball allowed mice to walk and run in one dimension (forward-reverse).

Stimuli were presented via an ED1 Electrostatic Speaker Driver and an ESI Electrostatic Speaker (TDT) positioned 4 inches directly in front of the animal. Experiments were performed in a sound attenuation chamber (Industrial Acoustics) at $65 \mathrm{~dB}$ SPL RMS measured at the animal's head using a sound level meter (Extech, model 407736) with A-weighting by normalizing to the SPL of an $8 \mathrm{kHz}$ reference tone. The speaker used has $\pm 11 \mathrm{~dB}$ output between 4 and $110 \mathrm{kHz}$. Stimuli consisted of broadband noise, four logarithmically spaced tones ranging between 4 and 32 $\mathrm{kHz}$, ultrasound noise bandpassed between 40 and $60 \mathrm{kHz}$, and 8 natural pup calls recorded from 2 to 4 postnatal day WT CBA/CaJ mouse pups, digitized at a sampling rate of $195.3 \mathrm{kHz}$. All stimuli were low pass filtered and amplified between the DAC and the speaker driver at $100 \mathrm{kHz}$ with a custom analog filter and preamp (Kiwa Electronics). Pup calls were recorded from pups isolated in a cage inside a sound attenuation chamber (Industrial Acoustics) with an ultrasound microphone (Avisoft) positioned $\sim 30 \mathrm{~cm}$ above the pup.

Single units were blindly recorded in vivo by the "loose-patch" technique using borosilicate glass micropipettes $(7-30 \mathrm{M} \Omega$ ) tip-filled with intracellular solution (125 mm potassium gluconate, $10 \mathrm{~mm}$ potassium chloride, 2 mm magnesium chloride, and 10 mm HEPES, pH 7.2). Spike 
activity was recorded using BA-03X bridge amplifier NPI Electronic Instruments), low-pass filtered at $3 \mathrm{kHz}$, digitized at $10 \mathrm{kHz}$, and acquired using Spike2 (Cambridge Electronic Design). The depth of the recorded cell was measured by a hydraulic manipulator (MX610, Siskiyou).

Photostimulation-assisted identification of PV cells. In addition to the head-bar implant, naive $A i 32^{+/-} ; P V-C r e^{+/-}$mice were implanted with an optic fiber (Ø105 $\mu \mathrm{m}$, NA 0.22 ; ThorLabs) pigtailed to a ceramic ferrule (Ø128 $\mu \mathrm{m}$ hole size; ThorLabs), stabilized with dental cement (Teets), and with the tip inserted into the left hemisphere of the auditory cortex. Stimuli consisted of trains of ten $10 \mathrm{~ms}$ pulses of blue light (473 $\mathrm{nm}$; OEM Laser) delivered at $2 \mathrm{~Hz}$, with power of 2.5-4.5 $\mathrm{mW}$ measured at the tip of the optic fiber.

Experimental design and statistical analysis. All data were manually spiked sorted into single-unit spike trains with Spike2 (Cambridge Electronic Design) and subsequently analyzed using custom software written in MATLAB (The MathWorks).

For photo identification of PV neurons (Lima et al., 2009), we computed the median latency over all trials to the first spike following light onset (trials with latency $>50 \mathrm{~ms}$ were set at $50 \mathrm{~ms}$ ), and the reliability as the proportion of trials where the cell produced at least 1 spike within 50 ms of light onset. Identified PV neurons exhibited latencies of a few ms and reliability close to 1. Peristimulus time histograms (PSTHs) in Figure $1 A$ depict spike probability in each bin as the number of spikes in all trials divided by the total number of trials. We identified the peak and trough of the mean spike waveform (from the spikes occupying the quartile from $70-95$ percentile amplitude) for every neuron reported in this study. We computed the interval between them and the ratio of the amplitudes of the trough and the peak. Based on our photo identification results, neurons with waveforms that had an interval of $\leq 0.5 \mathrm{~ms}$ and a trough/peak amplitude ratio of $>0.5$ were designated as pPV neurons. All other neurons were designated as putative non-PV neurons. Depth was recorded for all neurons: neurons at a depth of $<500 \mu \mathrm{m}$ were designated "superficial layer" and neurons at a depth of $>500 \mu \mathrm{m}$ were designated "deep layer." Data from neurons deeper than $1100 \mu \mathrm{m}$ were discarded.

We recorded from 14 pup naive WT females, 4 surrogate WT females, 13 pup naive $M e c p 2^{\text {het }}$ females, and 9 surrogate Mecp $2^{\text {het }}$ females for a total of 313 putative non-PV neurons and $66 \mathrm{pPV}$ neurons. In addition, we recorded from 8 pup naive Mecp $2^{\text {het }}$; Gad $1^{\text {het }}$ females, and 5 surrogate Mecp $2^{\text {het }}$; Gad1 $1^{\text {het }}$ females for a total of 61 putative non-PV neurons and 23 pPV neurons.

For the analysis in Figures 6, 7, and 9, we constructed a PSTH (10 ms bin size) of the mean spiking response across trials to each stimulus for each neuron. The bin values for the mean response to all stimuli were transformed to $Z$ scores. A bootstrap procedure was used to identify all individual cell-call pairs with a significant response to the call. Briefly, we randomly sampled 15 bins from all histograms for a given cell 10,000 times to create a null distribution, and we compared the mean bin value during the $150 \mathrm{~ms}$ after the stimulus. Responses that fell above the top $2.5 \%$, or below the bottom $2.5 \%$, of this distribution were considered significantly excitatory or inhibitory, respectively. Only significant responses were included in this analysis.

Response strength (as used in Figs. 3, 4, 5, and 8) was computed as the mean firing rate during the first $350 \mathrm{~ms}$ after stimulus onset, minus the mean firing rate during the preceding $3 \mathrm{~s}$ (which was also used to compute the reported spontaneous firing rate for each cell). Lifetime sparseness was calculated as follows (Meeks et al., 2010):

$$
S_{L}=\frac{1-\left\{\frac{\left(\sum_{j=1}^{N} \frac{\left|\Delta r_{j}\right|}{N}\right)^{2}}{\sum_{j=1}^{N} \frac{\left(\Delta r_{j}\right)^{2}}{N}}\right\}}{1-\frac{1}{N}}
$$

$N$ is the number of different stimuli presented to the neuron, and $\Delta r_{j}$ is the change in firing rate of the neuron in response to the $j$ th stimulus.
Statistical analysis was performed using parametric or nonparametric tests as noted in the text, with corrections for multiple comparisons where appropriate.

\section{Results}

Our previous work revealed molecular expression changes to inhibitory networks in the auditory cortex of surrogate female mice (Sur) that were cohoused with a mother and her pups beginning at their birth, compared with maternally inexperienced virgin females (Naive). We further showed that the changes to inhibitory circuitry were significantly altered in $M e c p 2^{\text {het }}$, and that independent genetic and pharmacological interventions that partially restored WT marker expression significantly improved behavioral performance (Krishnan et al., 2017). We speculated that the molecular expression differences between surrogate WT (SurWT) and Mecp $2^{\text {het }}$ (SurHET) might lead to or accompany differences in sensory activity in the auditory cortex. Therefore, we performed loose-patch extracellular recordings from individual neurons in the auditory cortex of awake, head-fixed female mice of both genotypes, before and after maternal experience.

\section{Photoidentification of PV neurons}

Previous studies have established that PV cortical neurons can be identified in electrophysiological recordings by the characteristics of their extracellular waveform (Wu et al., 2008; Oswald and Reyes, 2011; Cohen and Mizrahi, 2015). In one particularly relevant example, Cohen and Mizrahi (2015) made extracellular recordings from auditory cortical neurons under guidance by two-photon laser scanning microscopy. They reported that genetically labeled PV formed a cluster distinct from their non-PV pyramidal cell neighbors on a scatterplot of (1) the time between the positive peak of the spike waveform and the negative trough, and (2) the ratio of their amplitude.

We confirmed this finding in our recordings, using photoidentification (Lima et al., 2009) of PV neurons in a mouse line expressing Channelrhodopsin2 only in PV neurons (Fig. 1A). PV neurons were identified in recordings by their spiking response to blue light (473 nm), delivered through a light fiber placed next to the recording pipette. ChR2-expressing $\mathrm{PV}^{+}(n=9)$ were unambiguously identified by their highly reliable ( $>80 \%$ of trials) and short median latency $(<5 \mathrm{~ms})$ response to light (Fig. $1 B, C)$. Other cells recorded during the same experiments $(n=17)$ fired rarely to light ( $<25 \%$ of trials) and with very long median latencies $(>50 \mathrm{~ms})$, and they were deemed non-light-responsive and therefore likely non-PV (Fig. $1 B, C$ ).

Figure $1 D$ depicts a scatterplot of the peak-trough interval in milliseconds versus the trough/peak amplitude ratio for all WT and Mecp $2^{\text {het }}$ cells reported in this study $(n=379)$. The blue dots represent data points for photo-identified PV, and the red dots represent data points for cells that did not respond to light. Based on the segregation of optically identified PV to the quadrant of the plot bounded by peak-trough intervals $<0.6 \mathrm{~ms}$ and trough/ peak amplitude ratios $>0.5$, we designated all cells in that quadrant as pPV $(n=66)$ (Fig. $1 F)$. All other cells were designated as putative non-PV $(n=313)$ (Fig. $1 E)$, and likely are primarily pyramidal neurons (Cohen and Mizrahi, 2015). For simplicity and clarity, we henceforth refer to these putatively identified populations as "pPV" and "non-PV."

As is typically observed, we found that pPV neurons had higher spontaneous firing rates than non-PV cells across both genotypes and behavioral conditions (Fig. 2A; non-PV: 3.35 [3.0, 3.8] spikes/s; pPV: 7.50 [6.2, 8.7] spikes/s, Mann-Whitney U test; $p<0.001$ ) (all values reported as median and [95\% CI] through- 

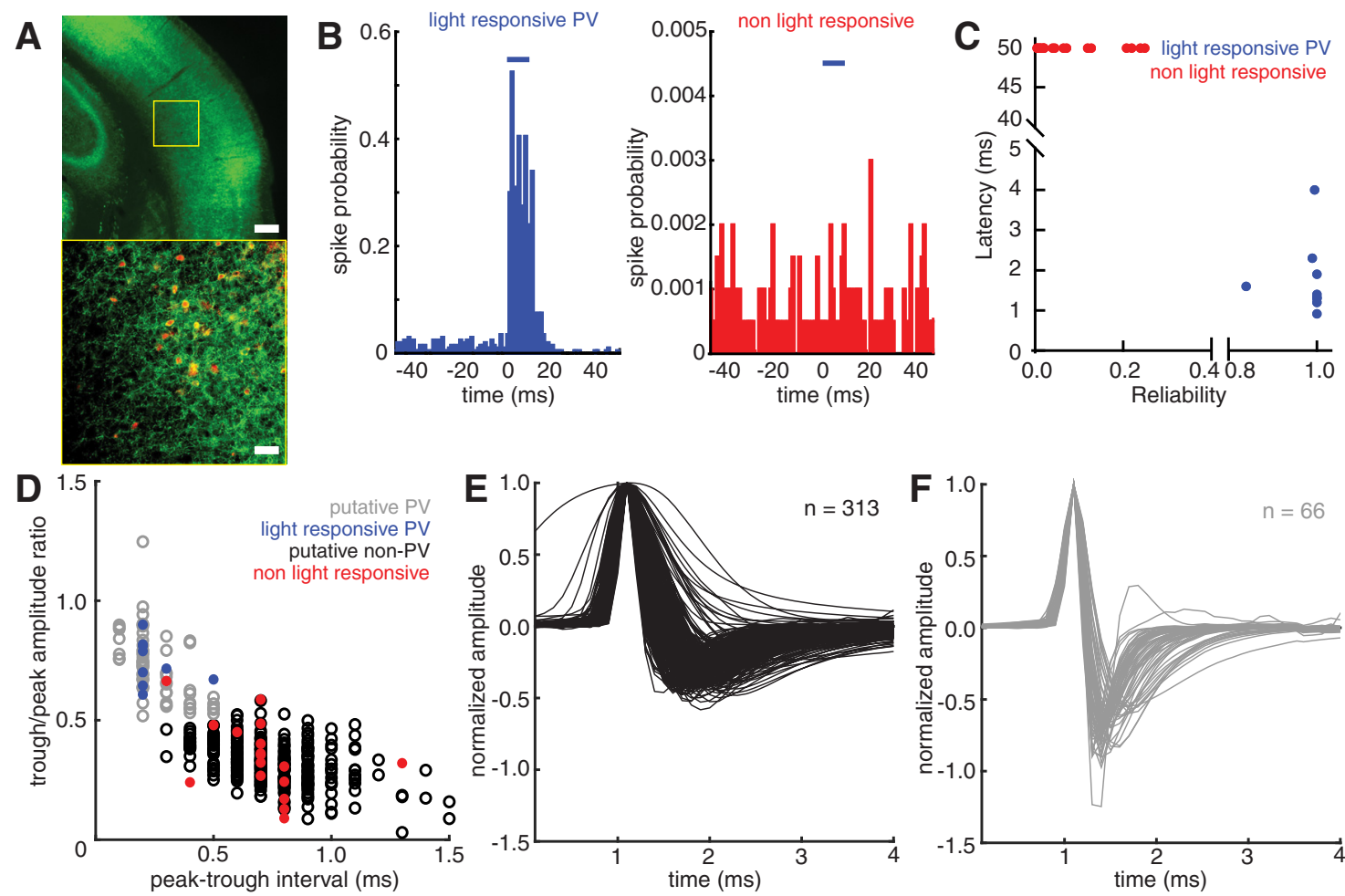

Figure 1. Optogenetic identification of PV neurons during in vivo neuronal recordings from auditory cortex in awake, head-fixed mice. $A$, Photomicrographs taken at $4 \times($ top) and $20 \times($ bottom) of a section of the auditory cortex in an Aiz2;PV-ires-Cre mouse expressing ChR2 in PV neurons. Green represents ChR2 fused to GFP. Top, Yellow box represents the location of the image in the bottom. Red represents PV. Scale bars: top, $200 \mu \mathrm{m}$; bottom, $50 \mu \mathrm{m}$. $\boldsymbol{B}$, Representative example histograms of responses in a positively identified PV neuron (left) and a presumed non-PV neuron (right) to $10 \mathrm{~ms}$ light pulses (blue bars, $473 \mathrm{~nm}$ ). Spike probability is computed as the number of spikes in each bin divided by the number of light trials. C, Scatterplot of the reliability of light responses (fraction of trials exhibiting spikes in response to light within the first $50 \mathrm{~ms}$ after light onset) versus the latency of spikes in response to light $(n=26)$. ChR2-expressing PV neurons ( $n=9$ ) are distinguished from non-ChR2-expressing presumed non-PV neurons $(n=17)$ by high reliability and low latency responses. $\boldsymbol{D}$, Scatterplot of spike waveform features for all neurons reported in this study $(n=379)$. The quantities plotted are the time in milliseconds between the peak and the trough of the averaged waveform (see Materials and Methods) versus the ratio of the amplitudes of the trough and the peak of the averaged waveform. Blue and red points represent the light-responsive and light-nonresponsive neurons shown in $\boldsymbol{C}$, respectively. Based on their locations in the scatterplot relative to the larger waveform dataset, the remaining neuronal waveforms were classified as pPV neurons (gray, $n=66$ ) and putative non-PV neurons (black, $n=313$ ). $\boldsymbol{E}, \boldsymbol{F}$, Plots of the averaged spike waveform from all putative non-PV $(\boldsymbol{E})$ and pPV $(\boldsymbol{F})$ neurons recorded for this study. Each waveform is aligned and normalized to its peak amplitude.
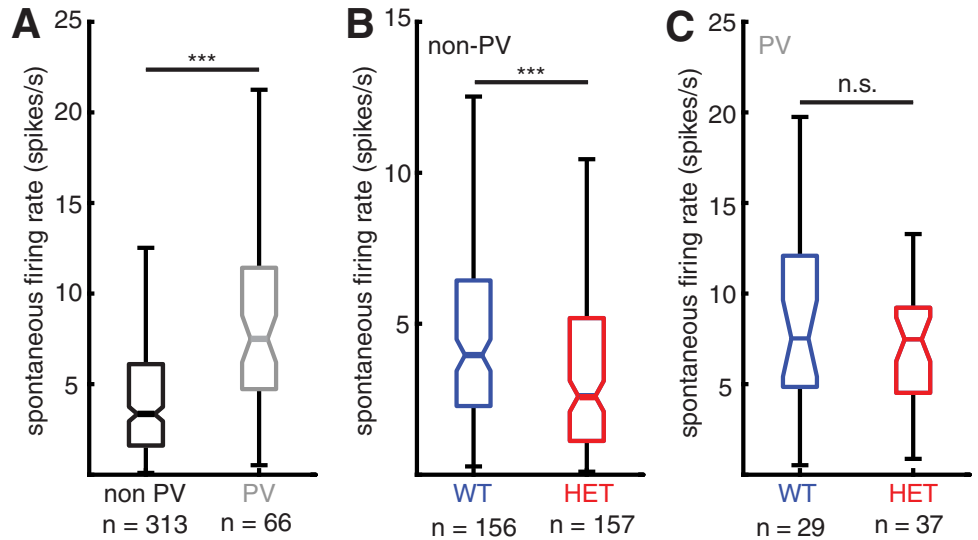

Figure 2. Spontaneous firing rates differ between cell types and genotypes. $A$, Boxplot of spontaneous firing rates comparing all non-PV neurons (black) and all pPV neurons (gray). ${ }^{* *} p<0.001$ (Mann-Whitney $U$ test). $\boldsymbol{B}$, Boxplot of spontaneous firing rates comparing all non-PV neurons from WT females (blue) and all non-PV neurons from HET females (red). ${ }^{* * *} p<0.001$ (Mann-Whitney $U$ test). C, Boxplot of spontaneous firing rates comparing all pPV neurons from WT females (blue) and all pPV neurons from HET females (red) (Mann-Whitney U test; n.s., Not significant, $p=0.54$ ).

out). Across behavioral conditions, non-PV neurons showed significantly lower spontaneous firing rates in $M e c p 2^{\text {het }}$ compared with WT (Fig. 2B; WT: 3.96 [3.4, 4.9] spikes/s; HET: 2.60 [2.0, 3.3] spikes/s; Mann-Whitney $U$ test, $p<0.001)$. Spontaneous firing rates did not differ between pPV neurons in WT and Mecp2 $2^{\text {het }}$
(Fig. 2C; WT: $7.53[5.2,11]$ spikes/s; HET: $7.48[5.8,8.6]$ spikes/s; Mann-Whitney $U$ test, $p=0.54)$. Spontaneous activity in neither cell type and neither genotype was affected by maternal experience $(p>$ $0.05)$.

\section{Responses to pup calls are strengthened} in SurWT, but not SurHET

We recorded auditory responses in the left "core" (thalamo-recipient) auditory cortex (Shepard et al., 2016) evoked by a library of eight different pup call exemplars recorded from a range of ages in our laboratory (Fig. 3A). We recorded from 18 $W T$ (14 naive and 4 surrogates) and 22 $M e c p 2^{\text {het }}$ (13 naive and 9 surrogates) for a total of 313 putative non-PV neurons and 66 pPV neurons. Individual neurons exhibited distinct responses to each exemplar. As a typical example, Figure $3 B$ shows raster plots and PSTHs for each call compiled from a recording of a single non-PV neuron. Some calls evoked firing rate suppression (inhibition), whereas others evoked firing rate increases (excitation). pPV neurons also typically exhibited distinct changes in firing rate to each call (Fig. $3 C$ ). 

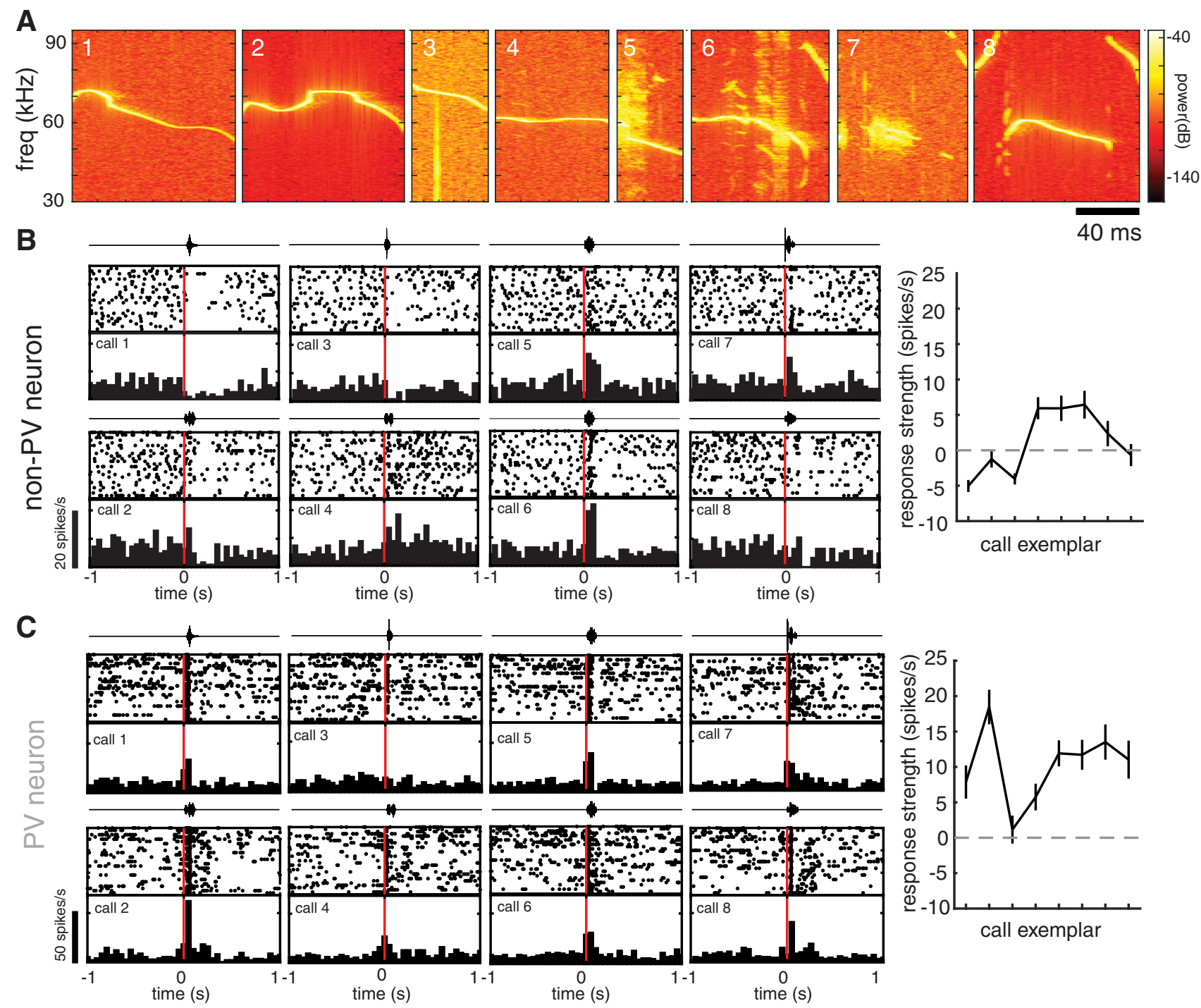

Figure 3. Individual auditory cortical neurons exhibit diverse responses to different pup call exemplars. $A$, Spectrograms of all call stimuli used in this study. $B, C$, Auditory responses of example non-PV (B) and pPV ( $\boldsymbol{C}$ neurons from naive WT females to a set of eight different pup call exemplars. Diverse responses to each of eight calls are depicted by a raster plot and PSTH (20 trials/call; bin size $=50 \mathrm{~ms}$ ). Above each raster and PSTH is the oscillograph of the call played during those trials. Right, Tuning curve plotting the mean change in firing rate during the first $200 \mathrm{~ms}$ after call onset (response strength). The use of the term "tuning curve" is in this case not intended to imply systematic neighbor relationships between stimuli on the $x$ axis.

As an initial assessment of maternal experience-dependent changes in neural encoding of pup calls, we examined the changes in median stimulus-evoked firing rates with maternal experience in each genotype. In $W T$, the median response strength (baselinesubtracted firing rate; see Materials and Methods) of non-PV cells to playback of pup calls was higher in SurWT than in NaiveWT (Fig. 4A; NaiveWT: $-0.28[-0.5,0.05]$ spikes/s; SurWT: $0.73[0.1,1.0]$ spikes/s; Mann-Whitney $U$ test, $p<$ 0.001 ). In contrast, SurHET showed a significant decrease in median pup call response strength compared with NaiveHET (Fig. 4A; NaiveHET: $0.37[0.1,0.7]$ spikes/s; SurHET: $-0.018[-0.2$, $0.1]$ spikes/s, Mann-Whitney $U$ test; $p=0.002$ ).

Our previous work (Krishnan et al., 2017) indicated that maternal experience triggers changes to inhibitory networks. Nevertheless, these changes in firing rate in response to call playback could, in principle, be due to either changes in excitatory or inhibitory input. Recordings from inhibitory pPV neurons suggest that the increase in auditory responses of non-pPV neurons in SurWT may be due to reduced inhibitory input from pPV neurons. Specifically, in $M e c p 2^{w t}$, increased call responses in non-PV cells of SurWT over NaiveWT was mirrored by significantly reduced call responses in pPV neurons (Fig. 4B; NaiveWT: 8.00 $[4.5,12]$ spikes/s; SurWT: 3.94 [1.2, 7.3] spikes/s; Mann-Whitney $U$ test; $p=0.007)$. On the other hand, no change in response strength was seen between NaiveHET and SurHET pPV neurons (Fig. 4B; NaiveHET: 4.82 [3.3, 6.0] spikes/s; SurHET: 4.19 [3.6, 7.0] spikes/s; Mann-Whitney $U$ test, $p=0.87$ ).

We next assessed whether the increase in pup call responses after maternal experience was layer-specific. We therefore sorted our recordings from non-PV cells according to depth, designating cells that were recorded $<500 \mu \mathrm{m}$ from the surface as superficial non-PV neurons and cells that were recorded at 500-1100 $\mu \mathrm{m}$ from the surface as deep non-PV neurons. Both $W T$ and $M e c p 2^{\text {het }}$ showed significant decreases in the median response strength of superficial non-PV neurons to pup calls following maternal experience (Fig. 4C; NaiveWT: $0.96[-0.7,3]$ spikes/s; SurWT: $-0.75[-2,1]$ spikes/s; Mann-Whitney $U$ test, $p=$ 0.014; NaiveHET: $1.61[0.57,3.9]$ spikes/s; SurHET: -0.19 $[-0.8,0.4]$ spikes/s; Mann-Whitney $U$ test, $p<0.001)$. However, increased pup call responses were observed in deep layer 

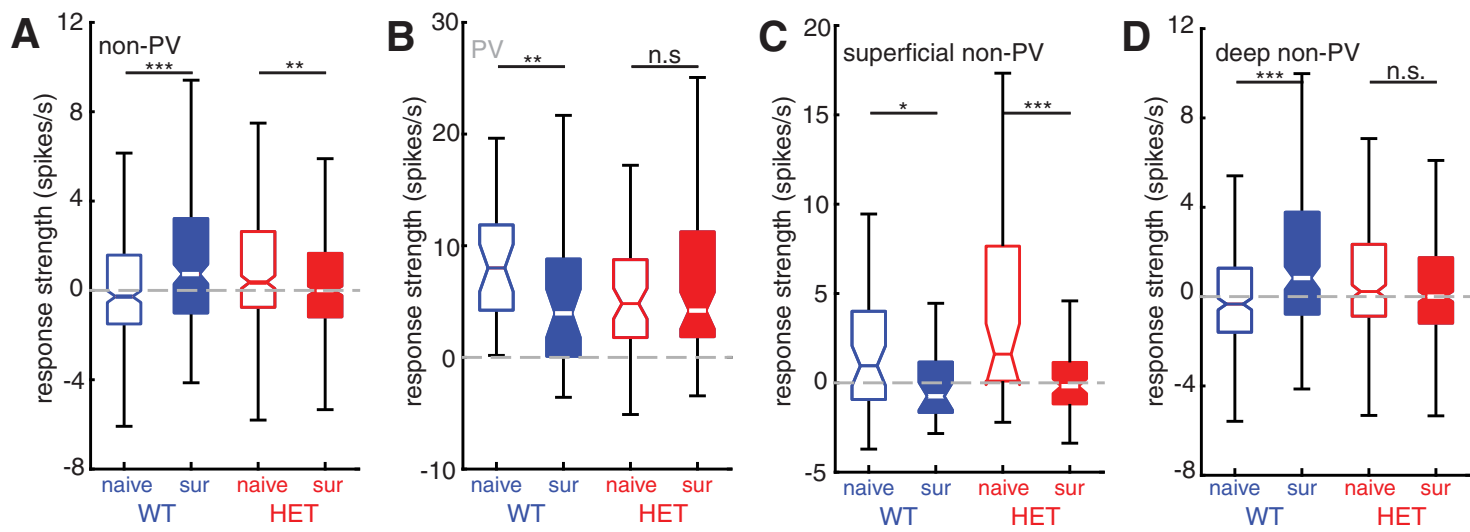

Figure 4. Maternal experience triggers distinct cell type- and layer-specific changes to pup call responses in WT and HET females. $A$, Boxplot of mean response strength to all calls for all non-PV cells comparing naive females with surrogate females for WT (blue; naive $n=344$ cell-call pairs and surrogate $n=224$ cell-call pairs) and $H E T$ (red; naive $n=344$ cell- call pairs and surrogate $n=456$ cell-call pairs) genotypes. Mann-Whitney $U$ test with Holm-Bonferroni correction: ${ }^{* * *} p<0.001 ;{ }^{* *} p=0.002 . B$, Boxplot of mean response strength to all calls for all pPV cells comparing naive females with surrogate females for WT (blue; naive $n=32$ cell-call pairs and surrogate $n=40$ cell-call pairs) and $H E T$ (red; naive $n=64$ cell-call pairs and surrogate $n=80$ cell-call pairs) genotypes. Mann-Whitney U test with Holm-Bonferroni correction: ${ }^{* *} p=0.007$; n.s., Not significant, $p=0.87$. C, Boxplot of mean response strength to all calls for all superficial (depth $<500$ $\mu \mathrm{m}$ ) non-PV cells comparing naive females with surrogate females for WT (blue; naive $n=48$ cell-call pairs and surrogate $n=32$ cell-call pairs) and HET (red; naive $n=48$ cell-call pairs and surrogate $n=80$ cell-call pairs) genotypes. Mann-Whitney $U$ test with Holm-Bonferroni correction: ${ }^{*} p=0.014 ;{ }^{* * *} p<0.001$. D, Boxplot of mean response strength to all calls for all deep (depth $<500 \mu \mathrm{m}$ ) non-PV cells comparing naive females with surrogate females for WT (blue; naive $n=296$ cell- call pairs and surrogate $n=192$ cell- call pairs) and HET (red; naive $n=296$ cell- call pairs and surrogate $n=376$ cell-call pairs) genotypes. Mann-Whitney $U$ test with Holm-Bonferroni correction: ${ }^{* * *} p<0.001$; n.s., Not significant, $p=0.07$.

non-PV in $W T$ (Fig. $4 D$; NaiveWT: $-0.33,[-0.6,-0.1]$ spikes/s; SurWT: $0.83[0.4,1.6]$ spikes/s; Mann-Whitney $U$ test, $p<$ 0.001), but not in Mecp $2^{\text {het }}$ (Fig. 4D; NaiveHET: 0.23 [ $\left.-0.05,0.6\right]$ spikes/s; SurHET: $0.00[-0.2,0.2]$ spikes/s; Mann-Whitney $U$ test, $p=0.07)$. Together, these results suggest that, in WT, auditory responses to pup calls, specifically in deep layer non-PV neurons, increase following maternal experience due to reduced inhibition by PV neurons. It is worth noting that, of the 27 callresponsive $\mathrm{PPV}$ neurons reported here, all but four were located in the deep layers. Considered in light of our previous work functionally implicating PV neurons in vocal perception behavior, these data also raise the possibility that plasticity in deep non-PV cells may be important for maternal behavioral learning, since it is disrupted in Mecp $2^{\text {het }}$.

\section{Plasticity of auditory responses in non-PV neurons is stimulus-specific}

Auditory responses of non-PV neurons to a sequence of synthetic and behaviorally irrelevant tone and noise stimuli were not affected by maternal experience in WT. Median response strength to synthetic stimuli did not differ in these neurons between $\mathrm{Na}$ iveWT and SurWT (Fig. 5A; NaiveWT: - $2.25[2.6,1.8]$ spikes/s; SurWT: $-2.23[-2.6,-2.0]$ spikes/s; Mann-Whitney $U$ test, $p=$ $0.84)$. pPV neuron responses to these stimuli were still significantly reduced in surrogates (Fig. 5B; NaiveWT: $0.21[-3.0,2.9]$ spikes/s; SurWT: $-3.7[-6.3,-2.1]$ spikes/s; Mann-Whitney $U$ test, $p=0.002$ ), but notably, the responses in non-PV were not affected. Potentially, this reflects a greater influence of PVmediated inhibition on non-PV neuron responses to pup vocalizations compared with synthetic sounds.

In $M e c p 2^{\text {het }}$, responses to the synthetic stimuli were affected by maternal experience in the same manner as responses to pup calls. Specifically, non-PV neurons in SurHET showed slightly, but significantly, lower firing rates than NaiveHET (Fig. 5A; NaiveHET: $-1.28[-1.8,-1.1]$ spikes/s; SurHET: $-1.92,[-2.2$, -1.7 ] spikes/s; Mann-Whitney $U$ test, $p=0.002$ ). In contrast to WT mice, median response strength of $\mathrm{pPV}$ neurons to synthetic stimuli was slightly but significantly increased between Naive-
HET and SurHET (Fig. 5B; NaiveHET: $-2.58[-4.5,-1.3]$ spikes/s; SurHET: $-0.90[-3.5,1.3]$ spikes/s; Mann-Whitney $U$ test, $p=0.032$ ).

\section{Plasticity of auditory responses in deep non-PV neurons affects the late response to calls}

We next analyzed the temporal structure of inhibitory and excitatory responses separately. In WT mice, many responses in both pPV and non-PV neurons exhibited biphasic structure, with an early component and a late component that appeared to be independently regulated by maternal experience. Stimulus onset and offset responses have been widely observed in auditory cortical neurons and are likely to arise from distinct synaptic inputs (Scholl et al., 2010). We found that, in WT, in addition to an overall shift of responses in favor of excitation, response plasticity specifically in the later part of the response effectively extended the excitatory responses of deep non-PV neurons.

First, we identified all cell-call pairs (mean response of one cell to one specific call) from deep layer non-PV neurons that resulted in a significant change in firing rate using a bootstrap test (see Materials and Methods). Then, the bins of all response PSTHs for each cell were normalized to a $Z$ score. Figure $6 A$ (top) depicts 2D PSTHs of all significant responses for each combination of genotype and maternal experience condition sorted from the most inhibitory responses to the most excitatory responses. Figure $6 \mathrm{~A}$ (bottom) represents the mean of all net excitatory and all net inhibitory responses comparing the magnitude and temporal structure between naive mice (black) and surrogate mice (red).

Qualitative comparison of the 2D PSTHs between NaiveWT and SurWT reveals that they are consistent with a shift in abundance and magnitude away from inhibitory responses and toward excitatory responses in SurWT. Comparing traces of the mean excitatory and inhibitory responses between NaiveWT and SurWT reveals that, distinct from the changes in abundance, there are time-dependent differences in the amplitude of responses. Specifically, we noticed that there are distinct changes early and late in the auditory responses, and these approximately 
corresponded to independent response phases seen in pPV neurons (see Fig. 7; and below). To quantify these differences, we integrated the area under the response curve during two time windows: 0-200 ms (early) and 200-350 ms (late), chosen to capture the timing of these response phases. The longest call stimulus was 125 $\mathrm{ms}$ in duration. The integrated area under the mean inhibitory response trace was significantly decreased in SurWT in both early (Fig. 6B; NaiveWT: 0.136 [0.13, 0.15] $Z$ scores $^{\star}$; S SurWT: 0.118 [0.10, $0.14] Z$ scores $^{\star}$ s; Mann-Whitney $U$ test, $p=0.004$ ) and late responses (Fig. 6C; NaiveWT 0.055 [0.04, 0.08] $Z$ scores $^{\star}$ s; SurWT: 0.039 [0.01, $0.06 Z$ scores $^{\star}$ s; Mann-Whitney $U$ test, $p=0.047$ ). This pattern is consistent with disinhibition of deep non-PV neurons throughout the response. Interestingly, the integrated area under the mean excitatory response trace was significantly increased in SurWT during the late response (Fig. $6 C$; NaiveWT: $0.010 \quad[0.0,0.03] Z$ scores $^{\star}$ s; SurWT: 0.050 [0.03, 0.07] $Z$ scores $^{\star}$ s; Mann-Whitney $U$ test, $p=0.005$ ), but not during the early response (Fig. $6 B$; NaiveWT: $0.223[0.17,0.27] Z$ scores $^{\star}$ s; SurWT: $0.181[0.14,0.24] Z$ scores $^{\star}$ s; Mann-Whitney $U$ test, $p=0.18$ ). Thus, auditory response plasticity in deep non-PV neurons, potentially reflecting removal of PV neuron-mediated inhibition, resulted in an extension of the excitatory response duration.

In contrast, we found no significant differences in the response integral in deep non-PV neurons between NaiveHET and SurHET during either the early response (Fig. 6D; NaiveHET excitatory: $0.203[0.17,0.23] Z$ scores $^{\star}$ s; SurHET excitatory: $0.220[0.19,0.26] Z$ scores $^{\star}$ s; Mann-Whitney $U$ test, $p=0.24$; NaiveHET inhibitory: $0.145[0.112,0.16] Z$ scores $^{\star}$ s; SurHET inhibitory: 0.143 [0.12, 0.15] $Z$ scores $^{\star}$ s; Mann-Whitney $U$ test, $p=0.97$ ) or the late response (Fig. $6 E$; NaiveHET excitatory: 0.013 [0.00, 0.02] Z scores $^{\star}$ s; SurHET excitatory: 0.027 [0.01, $0.04] Z$ scores $^{\star}$ s; Mann-Whitney $U$ test, $p=0.12$; NaiveHET inhibitory: $0.055[0.03,0.06] Z$ scores $^{\star}$ s; SurHET inhibitory: $0.052[0.03,0.06] Z$ scores $^{\star}$ s; Mann-Whitney $U$ test, $\left.p=0.75\right)$.

pPV neurons showed a complementary pattern of changes in their firing in the late portion of their response to pup calls. Figure $7 A$ (top) shows 2D PSTHs of pPV neuron responses in each genotype and maternal experience condition, and the bottom shows mean response traces comparing naive mice (black) to surrogate mice (red). In this case, because nearly all cells showed net excitatory responses, these traces represent the mean of all responses. Qualitatively, these panels reflect changes in pPV neuron output in the late response. As above, we quantified these changes by integrating the area under the response curve during
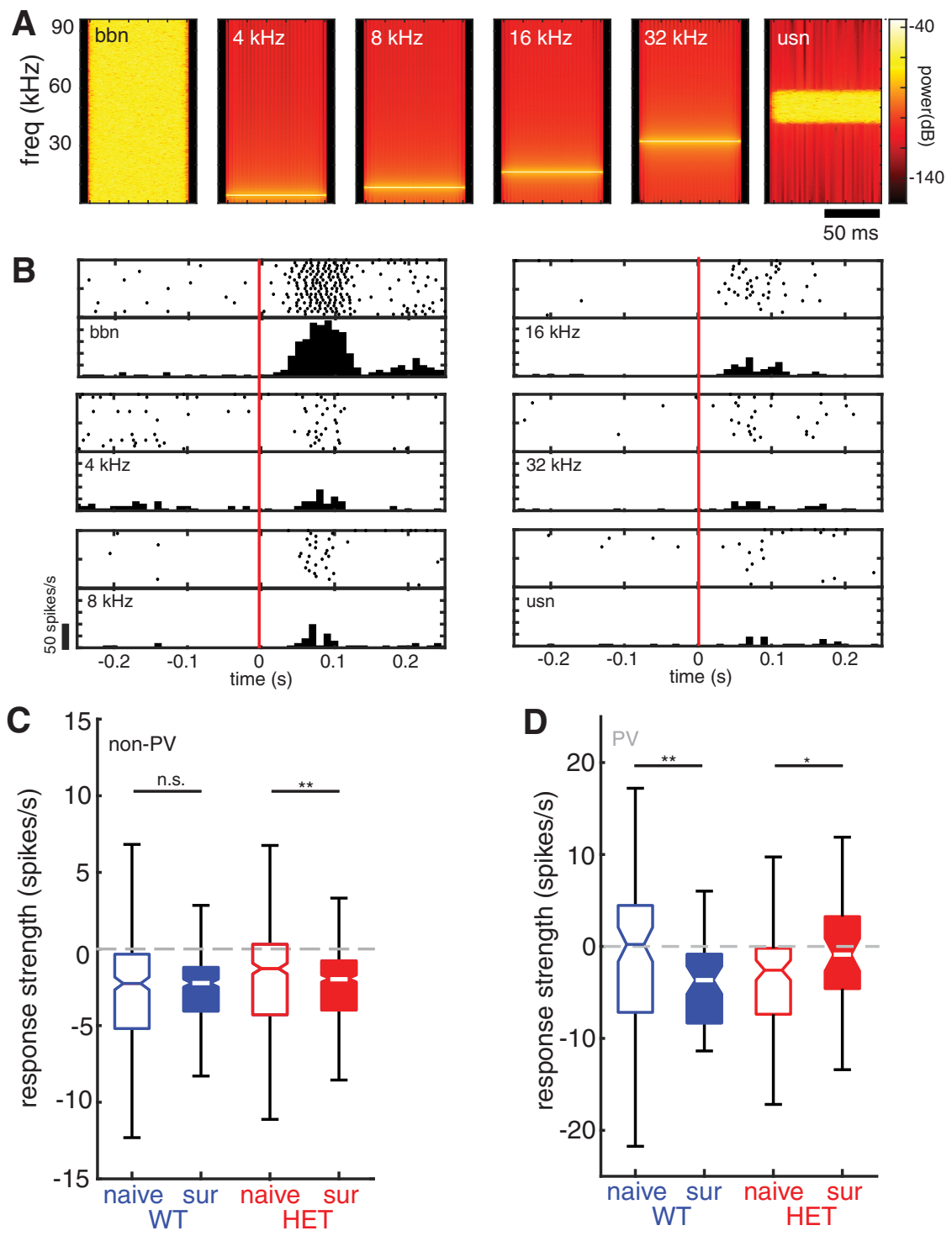

Figure 5. Maternal experience-dependent plasticity in WT females is stimulus-specific. $\boldsymbol{A}$, Spectrograms of all tones and noise stimuli used in this study. $\boldsymbol{B}$, Auditory responses of an example non-PV neuron from a naive WT female to each of the stimuli ( of mean response strength to tones and noise stimuli for all non-PV cells comparing naive females with surrogate females for WT (blue; naive $n=342$ cell-stimulus pairs and surrogate $n=288$ cell-stimulus pairs) and $H E T$ (red; naive $n=432$ cell-stimulus pairs icant, $p=0.84 ;{ }^{* *} p=0.002$. D, Boxplot of mean response strength to tones and noise stimuli for all $p P V$ cells comparing naive females with surrogate females for WT (blue; naive $n=96$ cell-stimulus pairs and surrogate $n=60$ cell-stimulus pairs) and HET (red; naive $n=108$ cell-stimulus pairs and surrogate $n=48$ cell-stimulus pairs) genotypes. Mann-Whitney $U$ test with HolmBonferroni correction: ${ }^{* *} p=0.002 ;{ }^{*} p=0.032$.

the early and late responses. In WT, pPV neurons showed a significant decrease in their response to calls during the late response (Fig. 7C; NaiveWT: $0.089[0.05,0.14] Z$ scores $^{\star}$ s; SurWT: $0.039[0.01,0.0] Z$ scores $^{\star}$ s; Mann-Whitney $U$ test, $\left.p=0.004\right)$, but not during the early response (Fig. $7 B$; NaiveWT: $0.166[0.12$, 0.16] $Z$ scores $^{\star}$ s; SurWT: $0.101[0.06,0.16] Z$ scores $^{\star}$ s; MannWhitney $U$ test, $p=0.09$ ). This is consistent with a model in which the increase in late responses of deep non-PV neurons described above in $W T$ is due to reduced firing in pPV neurons.

Suppression of PV-mediated inhibition was not observed in $M e c p 2^{\text {het }}$. Indeed, SurHET mice showed an increase in the strength of their late response to pup calls relative to NaiveHET (Fig. 7E; NaiveHET: $0.002[-0.02,0.03] Z$ scores $^{\star}$ s; SurHET: 

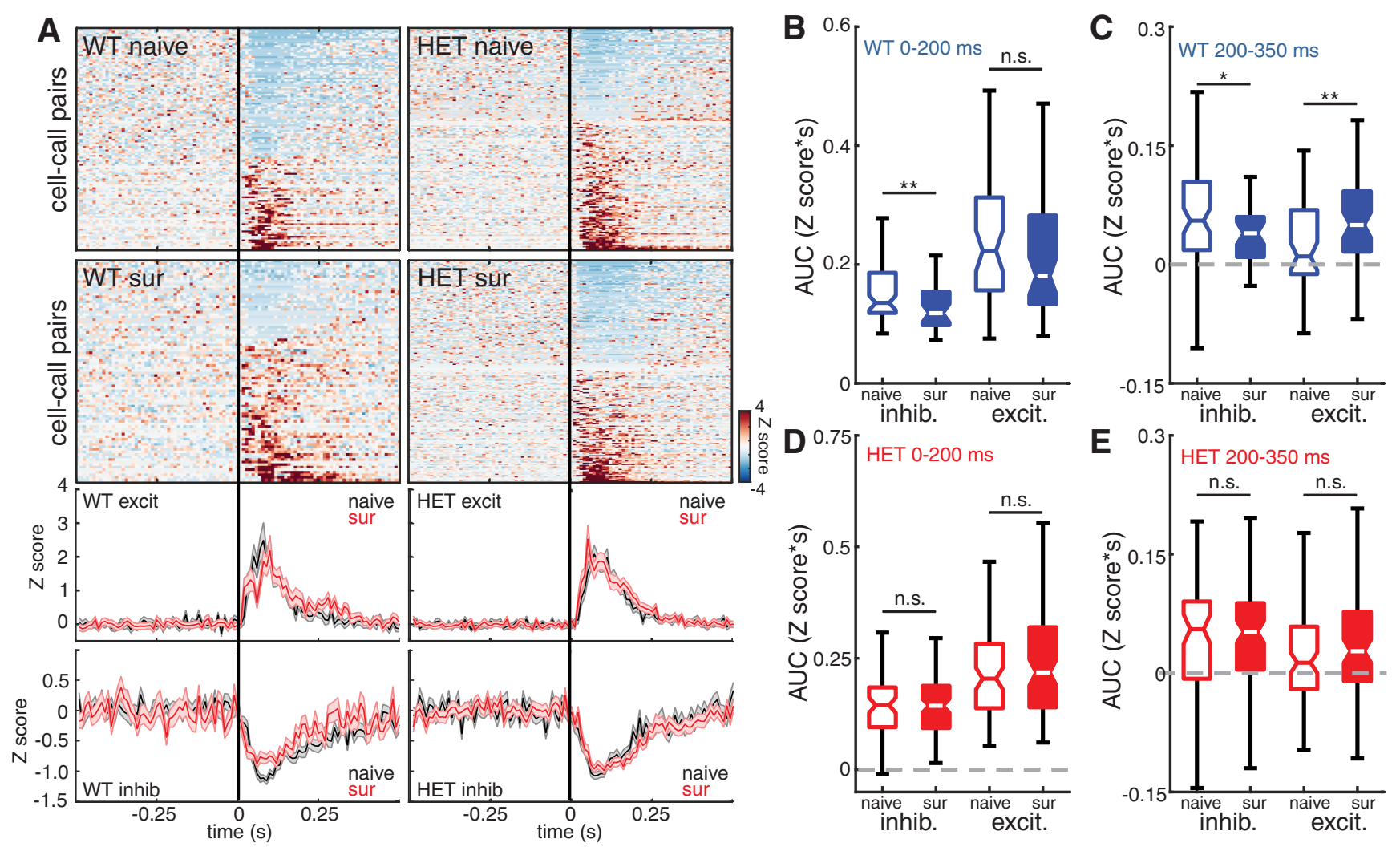

Figure 6. Excitatory responses of deep non-PV neurons to pup calls are disinhibited and extended in duration by maternal experience in WT females. $\boldsymbol{A}$, Top, Heat maps depicting Z scorenormalized responses for all deep non-PV cell-call pairs in naive and surrogate WT females and in naive and surrogate HET females. Each row in the heat map represents a PSTH of the mean response for a responsive cell-call pair (bin size $=10 \mathrm{~ms}$ ). Rows are sorted by response magnitude from most inhibitory to most excitatory. Bottom, Mean traces for excitatory and inhibitory cell-call pairs for each genotype comparing naive females (black) and surrogate females (red). Middle and outside lines indicate mean and SEM, respectively. $\boldsymbol{B}, \boldsymbol{C}$, Boxplot of the integrals (area under the curve $[A U C])$ of each inhibitory and excitatory response comparing naive and surrogate WT females. AUC was calculated for the early part of the response ( $0-200$ ms relative to stimulus onset). $\boldsymbol{B}$, Naive inhibitory: $n=103$; surrogate inhibitory: $n=51$; naive excitatory: $n=69$; surrogate excitatory: $n=67$. Mann-Whitney Utest with Holm-Bonferroni correction: ${ }^{* *} p=0.004 ; n . S .$, Not significant, $p=0.18$ and for the later part of the response ( $200-350$ ms relative to stimulus onset). $\boldsymbol{C}$, Mann-Whitney U test with Holm-Bonferroni correction: ${ }^{*} p=0.047 ;{ }^{* *} p=0.005 . \boldsymbol{D}, \boldsymbol{E}$, Boxplot of the integrals (AUC) of each inhibitory and excitatory response comparing naive and surrogate $H E T$ females. AUC was calculated for the early part of the response ( $0-200$ ms relative to stimulus onset). $D$, Naive inhibitory: $n=80$; surrogate inhibitory: $n=51$; naive excitatory: $n=116$; surrogate excitatory: $n=67$. Mann-Whitney $U$ test with Holm-Bonferroni correction: $n$.s., Not significant, $p=$ $0.41 ;$ n.S., Not significant, $p=0.29$; and for the later part of the response ( $200-350$ ms relative to stimulus onset). $\boldsymbol{E}$, Mann-Whitney U test with Holm-Bonferroni correction: $\mathrm{n} . \mathrm{S}$., Not significant, $p=0.75 ;$ n.s., Not significant, $p=0.12$.

$0.049[0.02,0.07] Z$ scores $^{\star}$ s; Mann-Whitney $U$ test, $\left.p=0.001\right)$. Early response strength was not changed (Fig. 7D; NaiveHET: $0.139[0.10,0.22] Z$ scores $^{\star}$ s; SurHET: $0.130[0.10,0.17] Z$ scores $^{\star}$ s; Mann-Whitney $U$ test, $\left.p=0.56\right)$.

\section{Maternal experience sharpens neuronal tuning for pup calls in WT}

We next assessed whether the auditory response plasticity we observed with maternal experience in deep non-PV neurons of WT coincided with changes in neural selectivity. We found that maternal experience led to stronger, sparser auditory responses and steeper tuning curves for pup vocalizations in $W T$, but not in $\operatorname{Mecp} 2^{\text {het }}$.

Figure $8 A$ shows a comparison of mean sorted tuning curves for all deep non-PV neurons from NaiveWT (black) and SurWT (red). The use of the term "tuning curve" here is not intended to imply systematic neighbor relationships between stimuli on the $x$ axis. Auditory responses were significantly stronger in SurWT than in NaiveWT for the most effective call stimuli (Mann-Whitney $U$ test with Holm-Bonferroni correction; $p<0.01)$. Consequently, the tuning curves in SurWT were steeper relative to NaiveWT. The same comparison for NaiveHET and SurHET showed that the mean sorted tuning curves from deep non-PV neurons in these two behavioral conditions (Fig. $8 B$ ) were indistinguishable (Mann-Whitney $U$ test; $p>0.05$ ). We also quantified and compared neural selectivity between naive and surrogate mice by computing lifetime sparseness for each neuron, a quantity that ranges from 0 (equal responses to all stimuli) to 1 (response to only one stimulus). Figure $8 C$ shows that maternal experience leads to a significant increase in mean lifetime sparseness for deep non-PV neurons in WT (NaiveWT: 0.255 [0.20, 0.23]; SurWT: 0.381 [0.29, 0.48]; Mann-Whitney $U$ test, $p<$ $0.001)$. In contrast, maternal experience did not significantly change lifetime sparseness for deep non-PV neurons in Mecp $2^{\text {het }}$ (NaiveHET: 0.312 [0.26, 0.45]; SurHET: 0.374 [0.31, 0.40]; Mann-Whitney $U$ test, $p=0.06$ ).

\section{Genetic reduction of GAD1 restores maternal behavior and pPV neuron plasticity}

Elimination of one copy of the gene GAD1 reduces expression of its protein product, the GABA-synthesizing enzyme GAD67, and also ameliorates overexpression of PV and PNNs in the developing visual cortex of male Mecp2-null mice (Krishnan et al., 2015). Remarkably, our previous work found that this manipulation of inhibitory networks not only reversed the increase in pPV and PNNs triggered by maternal experience in $M e c p 2^{\text {het }}$, but it also 

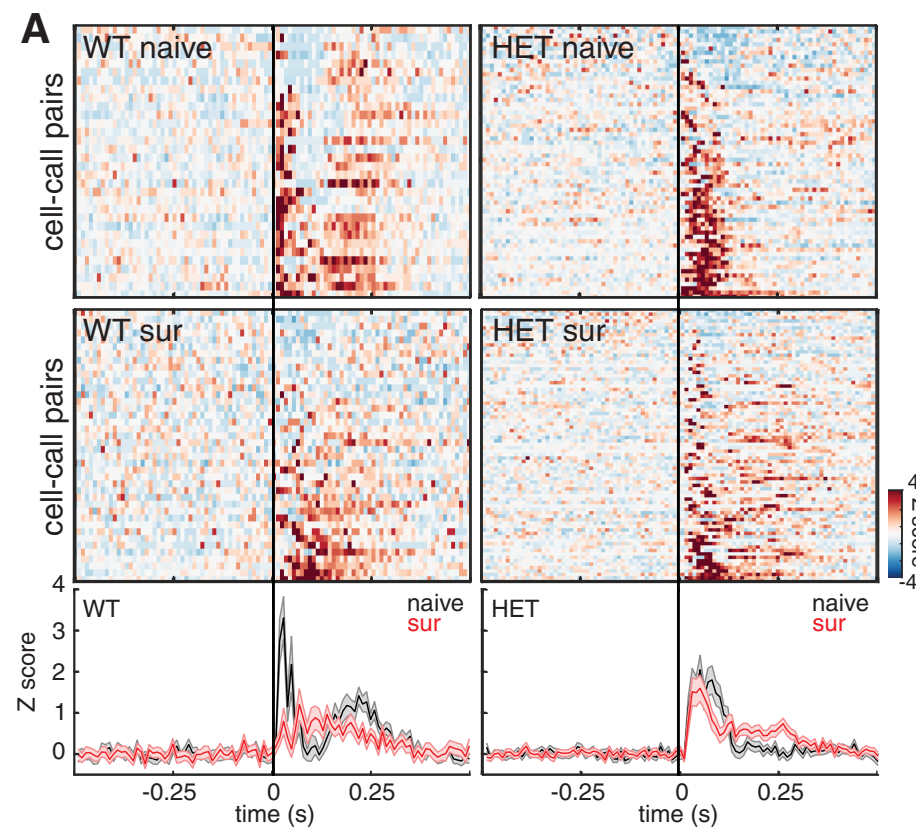
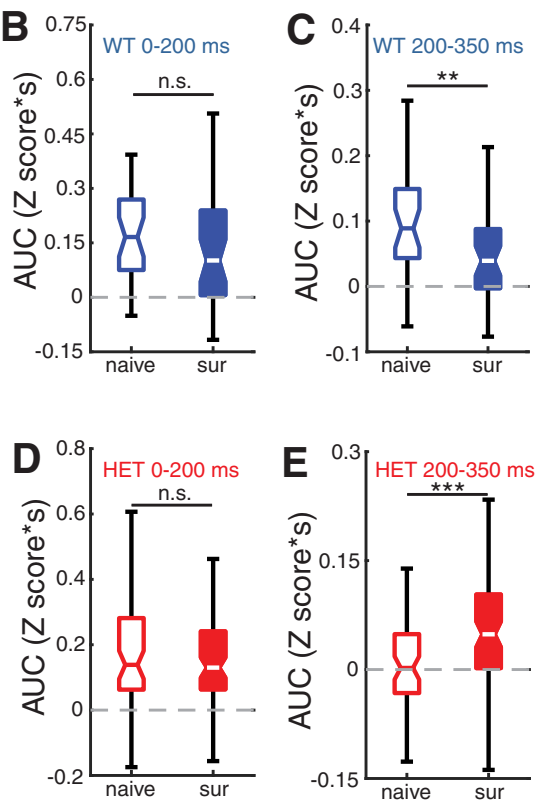

Figure 7. pPV neuron output is reduced after maternal experience in WT females and is strengthened after maternal experience in HET females. $\boldsymbol{A}$, Top, Heat maps depicting Z score-normalized responses for all pPV cell-call pairs in naive and surrogate WT females and in naive and surrogate HET females. Each row in the heat map represents a PSTH of the mean response for a cell-call pair (bin size $=10 \mathrm{~ms}$ ). Rows are sorted by response magnitude from least to most excitatory. Bottom, Mean traces for all cell-call pairs for each genotype comparing naive females (black) and surrogate females (red). Middle and outside lines indicate mean and SEM, respectively. $\boldsymbol{B}, \boldsymbol{C}$, Boxplot of the integrals (AUC) of each response comparing naive and surrogate WT females. AUC was calculated for the early part of the response ( $0-200$ ms relative to stimulus onset). $B$, Naive: $n=32$ neurons; surrogate: $n=40$ neurons. Mann-Whitney $U$ test; $n . S .$, Not significant, $p=0.09 ;$ and for the later part of the response (200-350 ms relative to stimulus onset). $\boldsymbol{C}$, Mann-Whitney $U$ test: ${ }^{* *} p=0.004$. D, E, Boxplot of the integrals (AUC) of each response comparing naive and surrogate HET females. AUC was calculated for the early part of the response ( $0-200$ ms relative to stimulus onset). $D$, Naive: $n=64$ neurons; surrogate: $n=80$ neurons. Mann-Whitney $U$ test; $n .5 .$, Not significant, $p=0.56$; and for the later part of the response ( $200-350$ ms relative to stimulus onset). $\boldsymbol{E}$, Mann-Whitney $U$ test: ${ }^{* * *} p=0.001$.
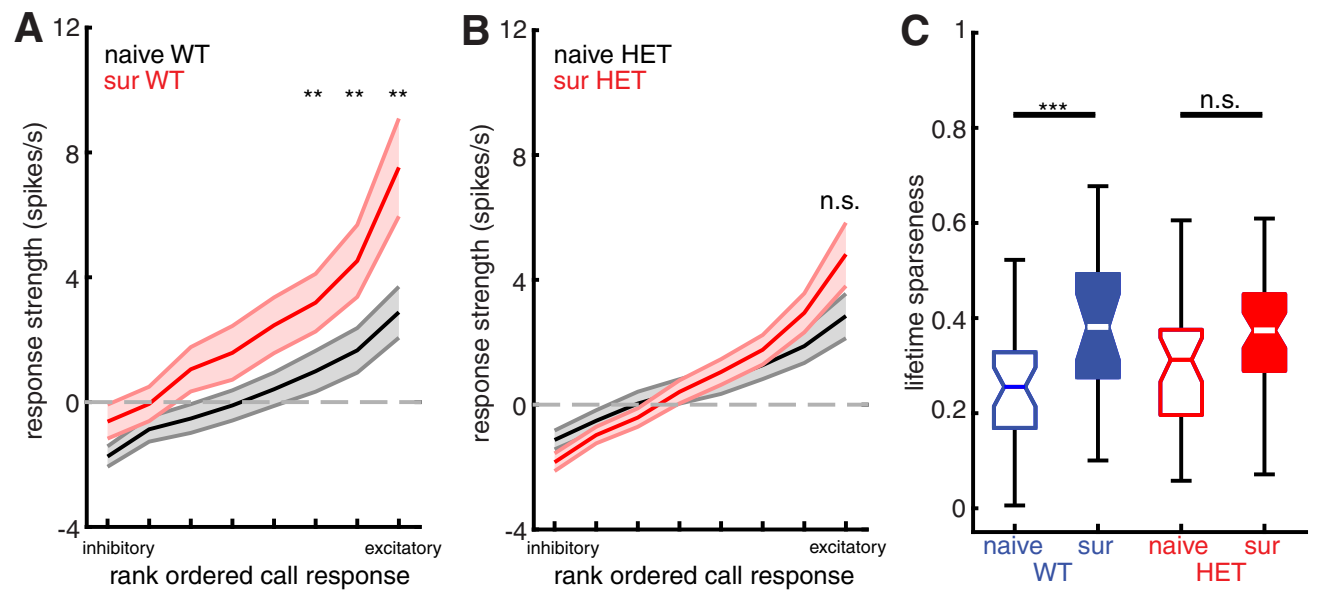

Figure 8. WT females show sharper tuning to calls in deep layer non-PV neurons after maternal experience, but HET females do not. $A$, Plot of mean sorted tuning curve for all deep layer non-PV neurons in WT females, comparing naive females (black) and surrogate females (red). Middle and outside lines indicate mean and SEM, respectively. Naive: $n=37$ neurons; surrogate: $n=24$ neurons. ${ }^{* *} p<0.01$ (Mann-Whitney $U$ test with Holm-Bonferroni correction). $\boldsymbol{B}$, Plot of mean sorted tuning curve for all deep layer non-PV neurons in HET females, comparing naive females (black) and surrogate females (red). Middle and outside lines indicate mean and SEM, respectively. Naive: $n=37$ neurons; surrogate: $n=47$ neurons. n.s., Not significant, $p>0.05$ (Mann-Whitney U test with Holm-Bonferroni correction). C, Boxplot of mean lifetime sparseness computed for each tuning curve from all deep non-PV cells comparing naive females with surrogate females for WT (blue; naive $n=37$ neurons and surrogate $n=24$ neurons) and HET (red; naive $n=37$ neurons and surrogate $n=47$ neurons) genotypes. ${ }^{* * *} p<0.001 ;$ n.S., Not significant, $p=0.06$ (unpaired $t$ test with Holm-Bonferroni correction).

restored pup retrieval proficiency to WT levels (Krishnan et al., 2017). Here we show that Mecp $2^{\text {het }}$; Gad ${ }^{\text {het }}$ exhibit partial reinstatement of maternal experience-induced plasticity of pPV neurons and enhanced firing of deep non-PV neurons in response to pup vocalizations.

We made loose-patch recordings from the left "core" auditory cortex of 13 female Mecp $2^{\text {het }}$; Gad1 ${ }^{\text {het }}$ mice (8 naive and 5 surrogates) for a total of 61 putative non-PV neurons and $23 \mathrm{pPV}$ neurons. Data collected in response to pup calls were analyzed as in Figures 6 and 7. Figure 9A (top) shows 2D PSTHs of non-PV neuron responses recorded in naive and surrogate Mecp $2^{\text {het }}$; Gad $1^{\text {het }}$ mice. Below are mean response traces comparing excitatory responses and inhibitory response for each group. In contrast to the Mecp $2^{\text {het }}$ only mice, the double mutants exhibited increased excitatory responses following maternal experience (Fig. 9B; NaiveHET; Gad1 ${ }^{\text {het: }} 0.131[0.11,0.16] Z$ scores $^{\star}$ s; 


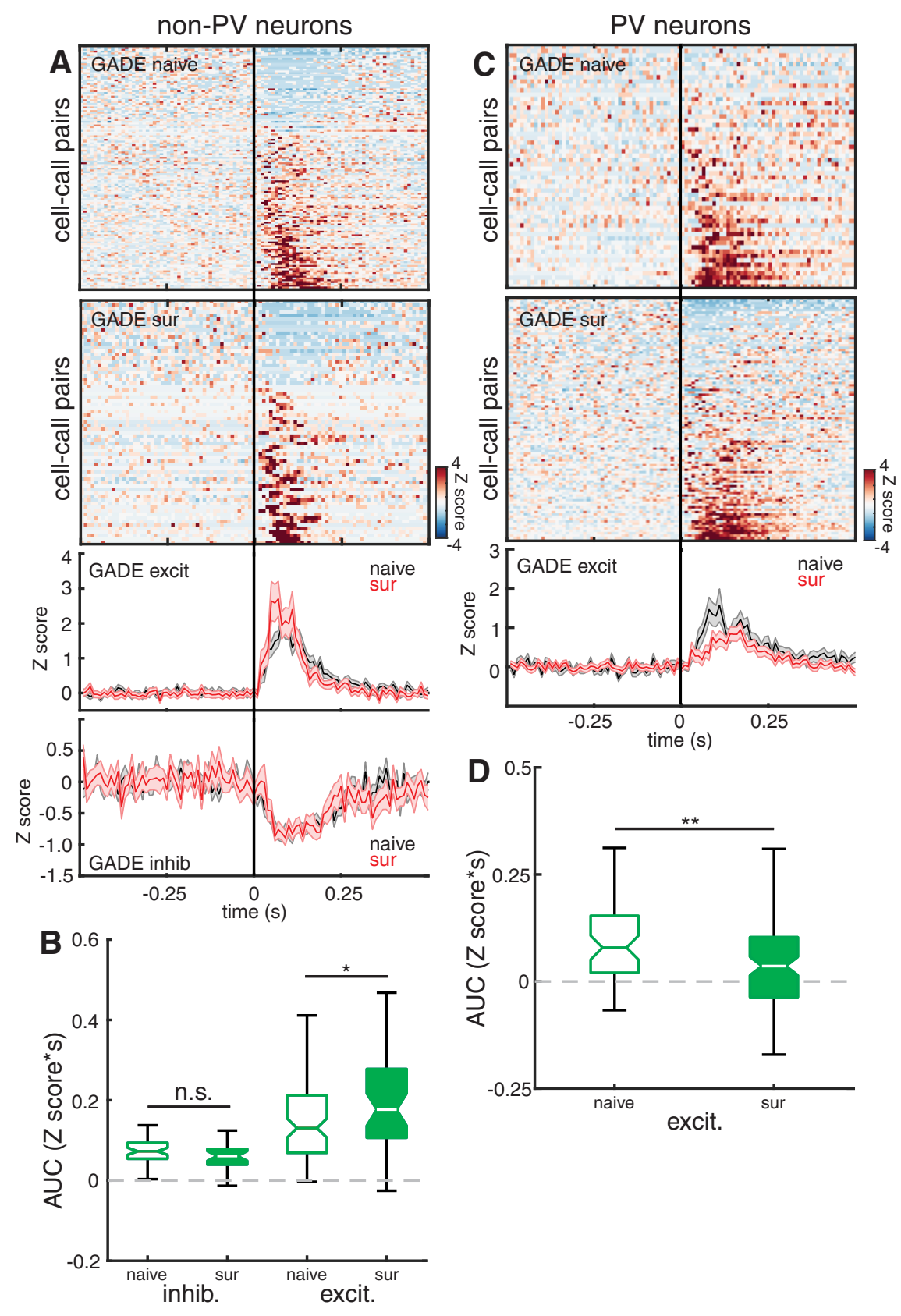

Figure 9. $\mathrm{pPV}$ neuron plasticity is restored in Mecp $2^{\text {het }}$; Gad $7^{\text {het }}$ mice. $A$, Top, Heat maps depicting Z score-normalized responses for all non-PV cell-call pairs in naive and surrogate Mecp2 $2^{\text {het }}$; Gad h het . Each row in the heat map represents a PSTH of the mean response for a cell-call pair (bin size $=10 \mathrm{~ms}$ ). Rows are sorted by response magnitude from least to most excitatory. Bottom, Mean traces for excitatory and inhibitory cell-call pairs for the double mutants comparing naive females (black) and surrogate females (red). Middle and outside lines indicate mean and SEM, respectively. B, Boxplot of the integrals (AUC) of each response comparing naive and surrogate $M e c p 2^{\text {het }} ; G a d 1^{\text {het }}$ females. AUC was calculated only for the activity during the stimulus $(0-120 \mathrm{~ms}$ relative to stimulus onset) (naive: $n=39$ neurons; surrogate: $n=22$ neurons). ${ }^{*} p=0.013$ (Mann-Whitney $U$ test). n.s., Not significant, $p=0.24$ (Mann-Whitney $U$ test). C, Top, Organized as in A, but data are from all pPV cells. Bottom, Mean traces for all cell-call pairs for each genotype comparing naive females (black) and surrogate females (red). Middle and outside lines indicate mean and SEM, respectively. D, Boxplot of the integrals (AUC) of each response comparing naive and surrogate Mecp $2^{\text {het; }}$ Gad $7^{\text {het }}$ females. AUC was calculated only for the activity during the stimulus $(0-120 \mathrm{~ms}$ relative to stimulus onset) (naive: $n=7$; surrogate: $n=16) .{ }^{* *} p=0.006$ (Mann-Whitney $U$ test).

SurHET; Gad1 ${ }^{\text {het }}$ : 0.177 [0.13, 0.24] $Z$ scores $^{\star}$ s; Mann-Whitney $U$ test, $p=0.013)$. This change differed from $W T$ plasticity in that it was only evident in the initial response during the stimulus (the first $120 \mathrm{~ms}$ ). Also, inhibitory responses in both conditions were small and did not significantly differ across behavioral conditions (Fig. 9B; NaiveHET; Gad1 ${ }^{\text {het }} 0.073[0.06,0.08] Z$ scores $^{\star}$ s;
SurHet; Gad1 ${ }^{\text {het: }} 0.062$ [0.04, 0.08] Z scores $^{\star}$ s; Mann-Whitney $U$ test, $p=$ 0.24). Nevertheless, recordings from $\mathrm{pPV}$ neurons in $M e c p 2^{\text {het }}$; Gad1 $1^{\text {het }}$ mice revealed reciprocal maternal experiencedependent changes, with lower spiking output of these inhibitory cells in surrogates in response to calls (Fig. $9 C, D ; \mathrm{Na}$ iveHet; Gad1 ${ }^{\text {het }}$ : $0.079[0.04,0.11] Z$ scores $^{\star}$ s; SurHet; Gad1 ${ }^{\text {het }}$ : 0.036 [0.01, 0.07] $Z$ scores $^{\star}$ s; Mann-Whitney $U$ test, $p=0.006)$. These results are consistent with partial restoration of the pattern of disinhibition observed in WT mice.

\section{Discussion}

Previously, we found that heterozygous female Mecp2 mutants failed to learn to accurately retrieve pups, and we showed that this was due to a requirement for MECP2 in the auditory cortex at the time of maternal learning (Krishnan et al., 2017). We also found that the absence of Mecp2 was linked to a rapid and inappropriate maturation of PV networks marked by overexpression of PV protein and PNNs. Maturation of PV networks is typically associated with the termination of plasticity, such as at the end of developmental critical periods, and the PV neuron population is therefore considered to be a "brake" on plasticity (Levelt and Hübener, 2012; Takesian and Hensch, 2013). Consistent with this framework, in our study, the accelerated development of $\mathrm{pPV}$ and PNNs and the behavioral deficit were reversed by genetic deletion of one copy of the gene Gad1, which codes for the GABA synthetic enzyme GAD67 (Krishnan et al., 2017).

Here we show that the precipitous maturation of the auditory cortical PV network in $M e c p 2^{\text {het }}$ after being exposed to pups for the first time is indeed correlated with impaired plasticity of neuronal responses specifically to vocal signals from pups. The reciprocal modulation of $\mathrm{pPV}$ and non-PV neurons strongly suggests that PV neurons in WT mice exert less inhibition of stimulus-evoked responses of deep non-PV presumptive pyramidal cells. Responses in the deep non-PV neurons are concomitantly sharpened to become more selective for pup calls. In contrast, in Mecp $2^{\text {het }}$, maternal experience-dependent changes in $\mathrm{PPV}$ neurons are blocked or even reversed. Consequently, no response plasticity and no increase in selectivity was observed in the deep non-PV population of SurHET. Loss of Mecp2 has been previously shown to abolish or interfere with short- and long-term synaptic plasticity and homeostatic plasticity (e.g., Asaka et al., 2006; Blackman et al., 2012; Na et al., 2013). Notably, deleting one Gad1 allele, which 

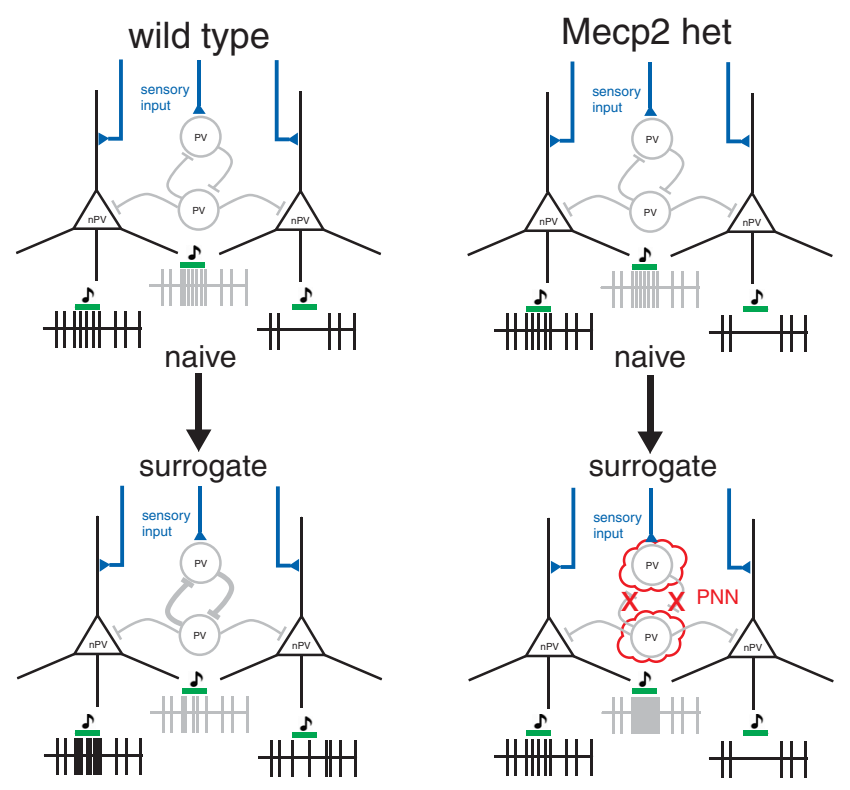

Figure 10. Proposed model for maternal experience-dependent plasticity in WT and Mecp $2^{\text {het }}$ mice. In NaiveWT mice, we propose that sensory input is received by PV and non-PV (nPV) neurons (in this simplified circuit, nPV neurons are assumed to be pyramidal neurons), and that spiking output from PV neurons (depicted by the gray spike train) attenuates excitatory responses and enhances inhibitory responses in the nPV neurons (depicted by the black spike trains). In the transition to surrogacy, PV neuron output is decreased, speculatively depicted here as occurring by stronger self-inhibition of the PV population, disinhibiting the nPV neurons. Consequently, in nPV neurons, weakly excitatory responses become stronger, and inhibitory responses become weaker. In Mecp $2^{\text {het }}$, PNNs interfere with synaptic plasticity at connections on PV neurons; thus, PV do not disinhibit the nPV cells.

we previously showed restores both inhibitory marker levels and behavioral performance (Krishnan et al., 2017), also leads to a partial restoration of pPV neuron plasticity and stronger excitatory responses in non-PV neurons. Together, our data are consistent with a model in which augmented pPV neuron maturation (Patrizi et al., 2019) in Mecp $2^{\text {het }}$ constrains neuronal plasticity in the auditory cortex. We speculate that this plasticity is an important component of early maternal learning. Although the Gad1 mutation is not specific to the auditory cortex, we argue that this pattern of results points to the auditory cortex as an important locus for the mutation to rescue behavior. The restoration of physiological plasticity by Gad1 KO here and the restoration of levels of inhibitory markers (Krishnan et al., 2017) represent independent confluent pieces of evidence that the Gad1 mutation may affect behavior by reversing the hypothetical pathology in auditory cortex. We also propose that altered PV networks in Mecp $2^{\text {het }}$ broadly impede plasticity during episodes of heightened learning in development or adulthood, thereby affecting other behaviors and circuits.

Our results show that maternal experience triggers a shift in the balance of excitatory and inhibitory responses to pup vocalizations exhibited by deep non-PV neurons. Specifically, the prevalence and magnitude of call-evoked firing suppression (inhibition) diminished in surrogates, while those of firing increases (excitation) grew. A parsimonious explanation for this shift is that the non-PV neurons become disinhibited following maternal experience. Indeed, inhibitory pPV neurons appeared to reciprocate with reduced spiking output, inviting us to speculate that they are the source of disinhibition in deep non-PV. We summarize the changes we observed after maternal experience, and we propose an explanatory circuit model (Figure 10).
In NaiveWT mice, we propose that sensory input is received by PV and non-PV neurons (Fig. 10). In this simplified circuit, non-PV neurons are assumed to be pyramidal neurons. Spiking output from PV neurons (depicted by the gray spike train) attenuates excitatory responses and enhances inhibitory responses in the non-PV neurons (depicted by the black spike trains). In the transition to surrogacy, PV neuron output is decreased, speculatively depicted here as occurring by stronger self-inhibition of the PV population, disinhibiting the non-PV neurons. Consequently, in non-PV neurons, weakly excitatory responses become stronger, and inhibitory responses become weaker. In Mecp $2^{\text {het }}$, PNNs interfere with synaptic plasticity at connections on PV neurons; thus, PV do not disinhibit the non-PV cells. We speculate that the lower levels of PNN expression in Mecp $2^{\text {het }}$;Gad1 $1^{\text {het }}$ mice liberate the PV network from that interference, thereby enabling its plasticity.

Interestingly, the disinhibition we see is stimulus-specific, affecting behaviorally significant calls but not synthetic sounds. On the other hand, decreased pPV spiking was still observed in response to synthetic sounds after maternal experience, suggesting that PV-mediated inhibition sculpts responses to vocalizations, but not simple tones and noise. At this time, it is unclear whether that is related to stimulus meaning or stimulus complexity. Moreover, the disconnect between the changes in pPV neurons versus non-PV neurons is consistent with changes in PV neurons leading those in non-PV neurons, as opposed to the other way around.

We also found that some aspects of the surrogacy-induced plasticity that we observed here were most prominent in the late phase of the response to calls. Interestingly, this observation bears similarity to a recent report that secondary auditory cortex (A2) neurons show maternal plasticity in offset responses to frequencymodulated stimuli (Chong et al., 2019). Nevertheless, we are confident that this effect does not bear on our data because our recordings were made in more dorsal thalamo-recipient auditory cortical areas, not in $\mathrm{A} 2$.

The changes in inhibition that we found are triggered by maternal experience may partially reflect and synergize with changes in auditory cortical inhibition seen in other studies. For example, Cohen and Mizrahi (2015) observed PV neuron-mediated disinhibition in layer $2 / 3$ of the auditory cortex when anesthetized lactating mothers were exposed to pup odors. Interestingly, this odor-induced form of disinhibition may be distinct from what we found here in deeper layers, which were not examined in that study. Marlin et al. (2015) found that the neuropeptide oxytocin, which plays a crucial role in the auditory cortex to establish maternal behavior, leads to acute disinhibition of responses to auditory stimuli, including pup calls. The long-term consequences of early maternal experience beyond weaning, however, may be increased inhibition, as Galindo-Leon et al. (2009) found evidence for in postweaning mothers.

Analysis of temporal patterns of firing in non-PV and pPV neurons revealed that both the disinhibition of non-PV cells and the suppression of firing in pPV cells were particularly evident late in the response to vocalizations. These effects synergized to effectively temporally extend responses of non-PV cells to pup calls. Disinhibition of auditory responses may be an important component in shaping neuronal selectivity for vocalizations. Disproportionate enhancement of the average responses to the preferred calls of individual neurons led to steeper tuning curves on average in surrogates. It may also have contributed to a significant increase in lifetime sparseness measured in SurWT compared with NaiveWT. The result of these changes extending 
response durations and increasing selectivity in deep cells that form the output of the auditory cortex may be a more sensitive representation of calls sent to downstream regions. This may be considered surprising because inhibition is classically believed to play a key role in sharpening selectivity of sensory responses; however, recent reexamination of this notion reveals more complexity (Wood et al., 2017). We anticipate this will be a focus of future work.

Nearly all the characteristic changes in pPV and non-PV neurons of WT were completely blocked in Mecp $2^{\text {het }}$ (Fig. 10). Indeed, we found that pPV neurons in Mecp $2^{\text {het }}$ showed even greater inhibitory output during the late response to vocalizations. These observations are completely consistent with the accelerated maturation of PV neurons we found in a previous study (Krishnan et al., 2017), which would be expected to increase the strength of inhibitory networks and arrest plasticity. We also used

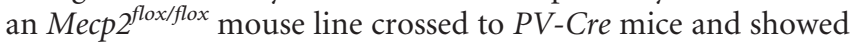
that $\mathrm{KO}$ of Mecp2 only in PV neurons was sufficient to impair maternal retrieval learning (Krishnan et al., 2017). Collectively, these observations strongly indicate that impaired PV neuron function is a central contributor to maternal behavior deficiencies in Mecp $2^{\text {het }}$.

Our data add to a body of evidence suggesting that maternal experience in adult nulliparous or primiparous female mice initiates an episode of heightened learning and plasticity that accesses mechanisms that overlap with those that control developmentally defined critical periods. It is well established that developmental critical periods, such as the visual cortical critical period, are regulated by a finely orchestrated sequence of removal, followed by reinstatement, of inhibition (Levelt and Hübener, 2012; Takesian and Hensch, 2013). The termination of these early critical periods is typically accompanied by maturation of intracortical inhibitory networks and intensification of PV expression and PNNs (Levelt and Hübener, 2012; Takesian and Hensch, 2013). We know that these events actively suppress network plasticity because dissolution of PNNs can actually reinstate visual cortical plasticity (Pizzorusso et al., 2002, 2006). More recent work further establishes that PV expression levels are inversely correlated with both neural plasticity and learning in adult mice (Donato et al., 2013).

Here we showed that, in WT, maternal experience leads to an enhancement of sensory responses in the auditory cortex that is likely implemented by decreased stimulus-evoked activity in the inhibitory pPV neurons. This pattern is shared with critical periods in development, raising the possibility that critical period mechanisms are reactivated in adulthood to facilitate learning. Therefore, early life plasticity mechanisms may be retained and accessed in adulthood, enabling more dynamic control of neural plasticity throughout life.

Our results are consistent with a broad base of evidence that Mecp2 specifically plays a role in regulating gene expression programs that govern plasticity during critical periods or other episodes of heightened learning (Picard and Fagiolini, 2019). One early indication of this came from the observation that Mecp2 regulates activity-dependent expression of BDNF (Chen et al., 2003; Zhou et al., 2006). Moreover, Mecp2 mutants exhibit accelerated critical periods for visual system development (Krishnan et al., 2015). In our own work, Mecp $2^{\text {het }}$ adult female mice showed similar expression levels of inhibitory markers in the auditory cortex as WT before pup exposure (Krishnan et al., 2017). The expression patterns diverged once the mice had acquired maternal experience. Thus, the learning triggered by social experience revealed aspects of $M e c p 2^{\text {het }}$ pathology that were previously la- tent. The idea that Mecp2 is more essential in developmentally or experientially defined windows is supported by nonlinear developmental trajectory of individuals with Rett syndrome. We propose based on our work that these observations may outline general principles of circuit pathology in Mecp2 mutation. Specifically, Mecp2 may act at different times and in different brain regions to regulate learning by modulating the threshold or capacity for circuit plasticity.

\section{References}

Agarwal N, Becker A, Jost KL, Haase S, Thakur BK, Brero A, Hardt T, Kudo S, Leonhardt H, Cardoso MC (2011) MeCP2 Rett mutations affect large scale chromatin organization. Hum Mol Genet 20:4187-4195.

Amir RE, Van den Veyver IB, Wan M, Tran CQ, Francke U, Zoghbi HY (1999) Rett syndrome is caused by mutations in X-linked MECP2, encoding methyl-CpG-binding protein 2. Nat Genet 23:185-188.

Asaka Y, Jugloff DG, Zhang L, Eubanks JH, Fitzsimonds RM (2006) Hippocampal synaptic plasticity is impaired in the Mecp2-null mouse model of Rett syndrome. Neurobiol Dis 21:217-227.

Atallah BV, Bruns W, Carandini M, Scanziani M (2012) Parvalbuminexpressing interneurons linearly transform cortical responses to visual stimuli. Neuron 73:159-170.

Banerjee A, Rikhye RV, Breton-Provencher V, Tang X, Li C, Li K, Runyan CA, Fu Z, Jaenisch R, Sur M (2016) Jointly reduced inhibition and excitation underlies circuit-wide changes in cortical processing in Rett syndrome. Proc Natl Acad Sci U S A 113:E7287-E7296.

Blackman MP, Djukic B, Nelson SB, Turrigiano GG (2012) A critical and cell-autonomous role for MeCP2 in synaptic scaling up. J Neurosci 32: 13529-13536.

Brink MC, Piebes DG, de Groote ML, Luijsterburg MS, Casas-Delucchi CS, van Driel R, Rots MG, Cardoso MC, Verschure PJ (2013) A role for $\mathrm{MeCP} 2$ in switching gene activity via chromatin unfolding and HPlgamma displacement. PLoS One 8:e69347.

Cardin JA, Carlén M, Meletis K, Knoblich U, Zhang F, Deisseroth K, Tsai LH, Moore CI (2009) Driving fast-spiking cells induces gamma rhythm and controls sensory responses. Nature 459:663-667.

Chao HT, Chen H, Samaco RC, Xue M, Chahrour M, Yoo J, Neul JL, Gong S, Lu HC, Heintz N, Ekker M, Rubenstein JL, Noebels JL, Rosenmund C, Zoghbi HY (2010) Dysfunction in GABA signalling mediates autismlike stereotypies and Rett syndrome phenotypes. Nature 468:263-269.

Chen WG, Chang Q, Lin Y, Meissner A, West AE, Griffith EC, Jaenisch R, Greenberg ME (2003) Derepression of BDNF transcription involves calcium-dependent phosphorylation of MeCP2. Science 302:885-889.

Chong KK, Dunlap AG, Kacsoh DB, Liu RC (2019) Experience-dependent coding of frequency-modulated trajectories by offsets in auditory cortex. bioRxiv 613448.

Cohen L, Mizrahi A (2015) Plasticity during motherhood: changes in excitatory and inhibitory layer $2 / 3$ neurons in auditory cortex. J Neurosci 35:1806-1815.

Cohen L, Rothschild G, Mizrahi A (2011) Multisensory integration of natural odors and sounds in the auditory cortex. Neuron 72:357-369.

Deng JV, Rodriguiz RM, Hutchinson AN, Kim IH, Wetsel WC, West AE (2010) $\mathrm{MeCP} 2$ in the nucleus accumbens contributes to neural and behavioral responses to psychostimulants. Nat Neurosci 13:1128-1136.

Deng JV, Wan Y, Wang X, Cohen S, Wetsel WC, Greenberg ME, Kenny PJ, Calakos N, West AE (2014) MeCP2 phosphorylation limits psychostimulant-induced behavioral and neuronal plasticity. J Neurosci 34: 4519-4527.

Donato F, Rompani SB, Caroni P (2013) Parvalbumin-expressing basketcell network plasticity induced by experience regulates adult learning. Nature 504:272-276.

Durand S, Patrizi A, Quast KB, Hachigian L, Pavlyuk R, Saxena A, Carninci P, Hensch TK, Fagiolini M (2012) NMDA receptor regulation prevents regression of visual cortical function in the absence of Mecp2. Neuron 76:1078-1090.

Ehret G, Koch M, Haack B, Markl H (1987) Sex and parental experience determine the onset of an instinctive behavior in mice. Naturwissenschaften $74: 47$

Galindo-Leon EE, Lin FG, Liu RC (2009) Inhibitory plasticity in a lateral band improves cortical detection of natural vocalizations. Neuron 62: 705-716. 
Gulmez Karaca K, Brito DVC, Zeuch B, Oliveira AMM (2018) Adult hippocampal $\mathrm{MeCP} 2$ preserves the genomic responsiveness to learning required for long-term memory formation. Neurobiol Learn Mem 149:84-97.

He LJ, Liu N, Cheng TL, Chen XJ, Li YD, Shu YS, Qiu ZL, Zhang XH (2014) Conditional deletion of Mecp2 in parvalbumin-expressing GABAergic cells results in the absence of critical period plasticity. Nat Commun 5:5036.

He Z, Hou W, Hao X, Dong N, Du P, Yuan W, Yang J, Jia R, Tai F (2017) Oxytocin receptor antagonist treatments alter levels of attachment to mothers and central dopamine activity in pre-weaning mandarin vole pups. Psychoneuroendocrinology 84:124-134.

Ito-Ishida A, Ure K, Chen H, Swann JW, Zoghbi HY (2015) Loss of MeCP2 in parvalbumin-and somatostatin-expressing neurons in mice leads to distinct Rett syndrome-like phenotypes. Neuron 88:651-658.

Kim H, Ährlund-Richter S, Wang X, Deisseroth K, Carlén M (2016) Prefrontal parvalbumin neurons in control of attention. Cell 164:208-218.

Krishnan K, Wang BS, Lu J, Wang L, Maffei A, Cang J, Huang ZJ (2015) $\mathrm{MeCP} 2$ regulates the timing of critical period plasticity that shapes functional connectivity in primary visual cortex. Proc Natl Acad Sci U S A 112:E4782-E4791.

Krishnan K, Lau BY, Ewall G, Huang ZJ, Shea SD (2017) MECP2 regulates cortical plasticity underlying a learned behaviour in adult female mice. Nat Commun 8:14077.

Lee SH, Kwan AC, Zhang S, Phoumthipphavong V, Flannery JG, Masmanidis SC, Taniguchi H, Huang ZJ, Zhang F, Boyden ES, Deisseroth K, Dan Y (2012) Activation of specific interneurons improves V1 feature selectivity and visual perception. Nature 488:379-383.

Levelt CN, Hübener M (2012) Critical-period plasticity in the visual cortex. Annu Rev Neurosci 35:309-330.

Lewis JD, Meehan RR, Henzel WJ, Maurer-Fogy I, Jeppesen P, Klein F, Bird A (1992) Purification, sequence, and cellular localization of a novel chromosomal protein that binds to methylated DNA. Cell 69:905-914.

Lima SQ, Hromádka T, Znamenskiy P, Zador AM (2009) PINP: a new method of tagging neuronal populations for identification during in vivo electrophysiological recording. PLoS One 4:e6099.

Lin FG, Galindo-Leon EE, Ivanova TN, Mappus RC, Liu RC (2013) A role for maternal physiological state in preserving auditory cortical plasticity for salient infant calls. Neuroscience 247:102-116.

Liu RC, Schreiner CE (2007) Auditory cortical detection and discrimination correlates with communicative significance. PLoS Biol 5:e173.

Marlin BJ, Mitre M, D’Amour JA, Chao MV, Froemke RC (2015) Oxytocin enables maternal behaviour by balancing cortical inhibition. Nature 520:499-504

Maxwell SS, Pelka GJ, Tam PP, El-Osta A (2013) Chromatin context and ncRNA highlight targets of MeCP2 in brain. RNA Biol 10:1741-1757.

McGraw CM, Samaco RC, Zoghbi HY (2011) Adult neural function requires MeCP2. Science 333:186.

Meeks JP, Arnson HA, Holy TE (2010) Representation and transformation of sensory information in the mouse accessory olfactory system. Nat Neurosci 13:723-730.

Miyata S, Kitagawa H (2017) Formation and remodeling of the brain extracellular matrix in neural plasticity: roles of chondroitin sulfate and hyaluronan. Biochim Biophys Acta Gen Subj 1861:2420-2434.

Morello N, Schina R, Pilotto F, Phillips M, Melani R, Plicato O, Pizzorusso T, Pozzo-Miller L, Giustetto M (2018) Loss of Mecp2 causes atypical synaptic and molecular plasticity of parvalbumin-expressing interneurons reflecting Rett syndrome-like sensorimotor defects. eNeuro 5:ENEURO. 0086-18.2018.
Na ES, Nelson ED, Kavalali ET, Monteggia LM (2013) The impact of MeCP2 loss- or gain-of-function on synaptic plasticity. Neuropsychopharmacology 38:212-219.

Noutel J, Hong YK, Leu B, Kang E, Chen C (2011) Experience-dependent retinogeniculate synapse remodeling is abnormal in MeCP2-deficient mice. Neuron 70:35-42.

Oswald AM, Reyes AD (2011) Development of inhibitory timescales in auditory cortex. Cereb Cortex 21:1351-1361.

Patrizi A, Awad PN, Chattopadhyaya B, Li C, Di Cristo G, Fagiolini M (2019) Accelerated hyper-maturation of parvalbumin circuits in the absence of MeCP2. Cereb Cortex pii: bhz085.

Picard N, Fagiolini M (2019) MeCP2: an epigenetic regulator of critical periods. Curr Opin Neurobiol 59:95-101.

Pizzorusso T, Medini P, Berardi N, Chierzi S, Fawcett JW, Maffei L (2002) Reactivation of ocular dominance plasticity in the adult visual cortex. Science 298:1248-1251.

Pizzorusso T, Medini P, Landi S, Baldini S, Berardi N, Maffei L (2006) Structural and functional recovery from early monocular deprivation in adult rats. Proc Natl Acad Sci U S A 103:8517-8522.

Scholl B, Gao X, Wehr M (2010) Nonoverlapping sets of synapses drive on responses and off responses in auditory cortex. Neuron 65:412-421.

Sewell GD (1970) Ultrasonic communication in rodents. Nature 227:410.

Shepard KN, Chong KK, Liu RC (2016) Contrast enhancement without transient map expansion for species-specific vocalizations in core auditory cortex during learning. eNeuro 3:ENEURO.0318-16.2016.

Sigal YM, Bae H, Bogart LJ, Hensch TK, Zhuang X (2019) Structural maturation of cortical perineuronal nets and their perforating synapses revealed by superresolution imaging. Proc Natl Acad Sci US A 116: 7071-7076.

Skene PJ, Illingworth RS, Webb S, Kerr AR, James KD, Turner DJ, Andrews R, Bird AP (2010) Neuronal MeCP2 is expressed at near histone-octamer levels and globally alters the chromatin state. Mol Cell 37:457-468.

Sohal VS, Zhang F, Yizhar O, Deisseroth K (2009) Parvalbumin neurons and gamma rhythms enhance cortical circuit performance. Nature 459:698-702.

Sorg BA, Berretta S, Blacktop JM, Fawcett JW, Kitagawa H, Kwok JC, Miquel M (2016) Casting a wide net: role of perineuronal nets in neural plasticity. J Neurosci 36:11459-11468.

Tai DJ, Liu YC, Hsu WL, Ma YL, Cheng SJ, Liu SY, Lee EH (2016) MeCP2 SUMOylation rescues Mecp2-mutant-induced behavioural deficits in a mouse model of Rett syndrome. Nat Commun 7:10552.

Takesian AE, Hensch TK (2013) Balancing plasticity/stability across brain development. Prog Brain Res 207:3-34.

Van den Veyver IB, Zoghbi HY (2000) Methyl-CpG-binding protein $2 \mathrm{mu}-$ tations in Rett syndrome. Curr Opin Genet Dev 10:275-279.

Wilson NR, Runyan CA, Wang FL, Sur M (2012) Division and subtraction by distinct cortical inhibitory networks in vivo. Nature 488:343-348.

Wood KC, Blackwell JM, Geffen MN (2017) Cortical inhibitory interneurons control sensory processing. Curr Opin Neurobiol 46:200-207.

Wu GK, Arbuckle R, Liu BH, Tao HW, Zhang LI (2008) Lateral sharpening of cortical frequency tuning by approximately balanced inhibition. Neuron 58:132-143.

Zhou Z, Hong EJ, Cohen S, Zhao WN, Ho HY, Schmidt L, Chen WG, Lin Y, Savner E, Griffith EC, Hu L, Steen JA, Weitz CJ, Greenberg ME (2006) Brain-specific phosphorylation of $\mathrm{MeCP} 2$ regulates activity-dependent BDNF transcription, dendritic growth, and spine maturation. Neuron $52: 255-269$. 